\title{
Label-free Lipidome Study of Paraventricular Thalamic Nucleus (PVT) of Rat Brain with Post-Traumatic Stress Injury by Raman Imaging
}

\author{
Ardalan Chaichi ${ }^{1}$, Syed Mohammad Abid Hasan ${ }^{1}$, Nishir Mehta ${ }^{1}$, Fabrizio Donnarumma ${ }^{2}$, Philip \\ Ebenezer $^{3}$, Kermit Murray ${ }^{2}$, Joseph Francis ${ }^{3}$, Manas Ranjan Gartia ${ }^{1, *}$ \\ ${ }^{1}$ Department of Mechanical and Industrial Engineering, Louisiana State University, Baton Rouge
} LA 70803

${ }^{2}$ Department of Chemistry, Louisiana State University, Baton Rouge LA 70803

${ }^{3}$ Comparative Biomedical Sciences, School of Veterinary Medicine, Louisiana State University, Baton Rouge LA 70803

*Corresponding author: mgartia@1su.edu 


\begin{abstract}
Post-traumatic stress disorder (PTSD) is a widespread psychiatric injury that develops serious life-threatening symptoms like substance abuse, severe depression, cognitive impairments and persistent anxiety. However, the mechanisms of post-traumatic stress injury in brain is poorly understood due to the lack of practical methods to reveal biochemical alterations in various brain regions affected by this type of injury. Here, we introduce a novel method that provides quantitative results from Raman maps in paraventricular nucleus of the thalamus (PVT) region. By means of this approach, we have shown a lipidome comparison in PVT regions of control and PTSD rat brains. Matrix-assisted laser desorption/ionization (MALDI) mass spectrometry was also employed for validation of the Raman results. Lipid alterations can reveal invaluable information regarding the PTSD mechanisms in affected regions of brain. We have showed that the concentration of cholesterol, cholesteryl palmitate, phosphatidylinositol, phosphatidylserine, phosphatidylethanolamine, sphingomyelin, ganglioside, glyceryl tripalmitate and sulfatide changes in the PVT region of PTSD compared to control rats. Higher concentration of cholesterol suggests the higher level of corticosterone in brain. ${ }^{1}$ Moreover, concentration changes of phospholipids and sphingolipids suggest the alteration of phospholipase A2 (PLA2) which is associated with inflammatory processes in the brain. Our results have broadened the understanding of biomolecular mechanisms for PTSD in PVT region of brain. This is the first report regarding the application of Raman spectroscopy for PTSD studies. This method has a wide spectrum of applications and can be applied to various other brain related disorders or other regions of brain.
\end{abstract}

Keyword: Raman Imaging; Raman Spectroscopy; Post-traumatic stress disorder; Paraventricular nucleus of the thalamus; Lipidome study; Lipidome quantification 


\section{Introduction}

Lipids play an important role in the brain through their homeostasis to maintain the integrity of the cell membranes and control the signaling through the membranes. ${ }^{2,3}$ Changes in brain lipids have been correlated with many neurological diseases ${ }^{4,5}$ such as Alzheimer's disease, ${ }^{6}$, ${ }^{7}$ Parkinson's disease, ${ }^{8,9}$ and schizophrenia. ${ }^{10,11}$ Structurally, brain tissues are composed of about $5-15 \%$ lipids that may account for $50 \%$ of the dry weight of the brain. ${ }^{12}$ Mass spectrometry has been the gold standard for lipidomics analysis and has been accurately applied to cells tissues and whole organisms. ${ }^{13-16}$ Most classical lipidome analysis requires extraction and homogenization of the sample, which results in a loss of spatial localization. Immunohistochemistry based methods preserve the spatial information. However, this is a targeted method and the target lipids must be known in advance to select an appropriate antibody for staining. Also, the number of dyes available for staining the lipids are limited. Further, multiplexing is difficult with the histochemical approach. For example, only one or two antibodies can be applied simultaneously on the same sample.

Both matrix-assisted laser desorption/ionization (MALDI) based mass spectrometry and Raman spectroscopy can alleviate many of the limitations listed above. Raman spectroscopy has additional advantages over MALDI such as Raman spectroscopy is non-destructive and no matrix is needed. In addition, Raman spectroscopy requires minimal sample preparation. Raman spectroscopy has become a ubiquitous method for molecular level analysis of various biological samples such as brain, ${ }^{17-20}$ heart, ${ }^{21-23}$ kidney, ${ }^{24-26}$ lipids, ${ }^{27,} 28$ and proteins ${ }^{29-31}$ due to the noninvasive and label-free nature of it. Among these, Raman spectroscopy of lipids has attracted particular attention in the field because of the strong Raman scattering of lipids provided by long nonpolar acyl chains in their structure. ${ }^{27}$ It is well-established that lipids play significant role in 
different cellular functions such as transport in cell membranes, signaling and energy storage. ${ }^{32}$ Therefore, the Raman signal obtained from the lipid bands of cells could be utilized as pathological biomarkers. Although there are similarities in the Raman spectrum of different lipids, individual lipids possesses unique spectra depending on numerous factors including geometry, phase, solubility, saturation, and polymorphism. ${ }^{33}$ Lipids have Raman bands in both the fingerprint (400$\left.1800 \mathrm{~cm}^{-1}\right)$ and higher wavenumber group frequency regions $\left(2400-3800 \mathrm{~cm}^{-1}\right){ }^{34}$ The most typical characteristics of lipids which originate from hydrocarbon chains manifest themselves in $1200-1050 \mathrm{~cm}^{-1}$ (C-C stretch), $1250-1300 \mathrm{~cm}^{-1}\left(\mathrm{CH}_{3}\right.$ scissor and twist) and $1400-1500 \mathrm{~cm}^{-1}\left(\mathrm{CH}_{2}\right.$ scissor and twist) ranges. ${ }^{35}$ At higher wavenumbers, strong Raman bands appear in the 2800-3100 $\mathrm{cm}^{-1}$ region which is assigned to $\mathrm{C}-\mathrm{H}$ stretching of lipids. ${ }^{27}$

Post-traumatic stress disorder (PTSD) is considered a prevalent psychiatric disorder caused by exposure to repeated or single life-threatening events such as individuals involved in traffic accidents, combat veterans and rape victims.$^{36}$ PTSD patients relive traumatic event by sudden remembrance of traumatic memories or flashbacks originating from the extreme horror and feelings of helplessness caused by the traumatic event. People with PTSD develop psychiatric disorders and symptoms such as severe depression, substance abuse, cognitive impairments, and persistent anxiety ${ }^{37}$ Controlled biological investigations of PTSD in human subjects are mostly restricted due to ethical and logistical issues. Thus, indirect neurobiology studies on post-traumatic stress injured brains have become conventional by means of translational animal model approaches. ${ }^{38,39}$ As a result, many studies have been carried out by exposing animals to acute or chronic stress conditions to study their physiology and behavior changes which provide valuable knowledge under conditions similar to that experienced by traumatized human subjects. Although traumatic memories can develop into PTSD, it is not the only influential factor involved in 
constructing an effective animal model due to multidimensional nature of this disease. ${ }^{40}$ Accordingly, various animal models such as predator stress, inescapable shocks, single prolonged stress, and unpredictable variable stress have been developed to ensure the occurrence of severe fear stress and production of human-like biological and behavioral symptoms in animals. ${ }^{41}$

It is well-known that severe stress exposure negatively affects various parts of brain which are responsible for emotional responses, memory, and decision-making functions. ${ }^{42-45}$ Numerous morphological and functional deteriorations in various regions of their brain including the hippocampus, prefrontal cortex, amygdala and thalamus have been observed in animal models exposed to prolonged stress conditions. ${ }^{42,46}$ Since physiological and behavioral symptoms of PTSD in brain and their connection is crucial to thoroughly comprehending this type of disorder. However, most of the studies in this field have been directed toward the hippocampus and prefrontal cortex regions and the other parts are frequently neglected due to complexity. ${ }^{47-50}$ The paraventricular nucleus of the thalamus (PVT) is one of the stress sensors in mammalian brains that has been recently examined for its correlation to post-traumatic stress disorders. ${ }^{51}$ According to recent studies, ${ }^{52,53}$ both psychological and physical stressors can affect and activate this region. Meanwhile, the correlation between adaptive behavioral responses due to severe stress and PVT region of brain is still tangled. PVT is a part of the thalamic nuclei located at the midline and intralaminar region and it is commonly assumed to participate in the arousal system. ${ }^{51}$ According to neuroanatomical investigations, ${ }^{51-53}$ PVT collects autonomic and arousal projections of the brainstem and nervous system. These studies have experimentally demonstrated PVT activation by arousal and stress stimulators. Furthermore, it was recently shown that drug addiction behaviors are also associated with this region of brain. ${ }^{54}$ In addition, substance addiction projections to the prefrontal cortex, amygdala, and nucleus accumbens are likely to originate from the PVT region. ${ }^{55}$ 
Despite the large population of patients affected by PTSD worldwide, there are no available reports regarding the application of Raman spectroscopy or imaging for this type of brain disorder. In order to understand the biochemical effects of PTSD on different regions of the brain which are controlling vital functions, it is substantially important to monitor the lipid alterations in those regions. ${ }^{32}$ Accordingly, novel therapeutic approaches can be developed to suppress these stressinduced changes. Recent studies illustrate the importance of stress-induced lipid metabolism analysis and their huge impact on unravelling the functions of brain regions. ${ }^{3,56}$ As a tangible example, it has been reported that prolonged stress stimulates an important lipid enzyme (phospholipase A2) which directly influences inflammatory responses by altering cellular lipid signaling. ${ }^{57}$ Furthermore, stress-induced lipid modulations affect the PVT region by altering arachidonoylglycerol and diacylglycerol levels. ${ }^{46}$ Additionally, ceramide level changes have also been observed in the brain due to prolonged stress conditions. ${ }^{57}$

There is a growing interest in discovering novel therapeutics for the treatment and molecular imaging methods for PTSD. We present new findings regarding the use of Raman spectroscopy imaging for measuring lipid changes in the brain. Specifically, we describe an approach for analyzing changes in the lipid levels in the PVT region of the brain tissue by obtaining 2D images of the formalin fixed brain sections from PTSD induced and control rats. We have used both unbiased PCA analysis and targeted approaches using lipid standards to identify lipid classes. We have validated the Raman imaging results with MALDI MS data. We anticipate that similar studies can be accomplished in other regions of brain to better understand the impact of stressinduced modulations on behavioral and physiological responses in PTSD.

\section{Materials and Methods}

\subsection{Biochemicals and Reagents}


To build a Raman reference library, purified lipid standards such as phosphatidylinositol (P66361G), phosphatidylserine (P7769-25MG), phosphatidylethanolamine (P1348-25MG), cholesteryl palmitate (C6072-1G), cholesterol (C8667-1G), galactocerebroside (C4905-10MG), glyceryl tripalmitate (T5888-1G), phosphatidic acid (P4013-100MG) and sphingomyelin (S0756-100MG) were purchased from Sigma-Aldrich (St. Louis, Missouri). Other lipids such as sulfatide (Avanti \# 131305) and ganglioside (Avanti \# 860053) were purchased from Avanti Polar Lipids (Alabaster, Alabama).

\subsection{Brain Tissue Sample Preparation}

Naive adult male Sprague-Dawley rats $(\mathrm{n}=12)$ (Harlan Laboratories, Indianapolis, $\mathrm{IN})$ weighing 325-350 $\mathrm{g}$ with age of 10 weeks were used for this study. The animals were fed $a d$ libitum and kept under standard laboratory conditions (temperature: $20^{\circ} \mathrm{C}$, humidity: $23-42 \%$ ). Alternating dark and light cycles (lasting $12 \mathrm{~h}$ each) was maintained. All animal handling procedures was approved by the Institutional Animal Care and Use Committee (IACUC) of the Louisiana State University School of Veterinary Medicine.

We used an acute predator exposure model ${ }^{38,58-61}$ to induce traumatic stress in the animal. In this model, rats were exposed periodically to a cat (adult, 7 years old, Harlan Laboratories, Indianapolis, IN) followed by rotating the rats into different cages to eliminate any social support and to induce chronic psychological stress. The experiments continued for 31 days and the rats were exposed to the cat on day 1 and day 11 for $1 \mathrm{~h}$. The first exposure was performed during the daylight cycle (07:00 - 19:00), while the second exposure was performed during the night cycle (19:00 - 07:00). Between Day 1 to Day 31, the rats were subjected to random cage rotation to make sure that no rat was housed with the same set of rats on consecutive days or more than four times within the experimental period. Also, it is important to note that during the exposure period, 
the cat was not allowed to touch the rats by putting the rats in Plexiglas containers. Further, during the cage rotation periods care was taken not to allow any cat or cat material near the cages. The control group $(n=6)$ were not subjected to exposure to cat or cage rotation and were kept in the same cages from Day 1-Day 31.

The behavioral test for anxiety was performed on all the rats using an elevated plus maze (EPM) experiment (EB-Instruments, Bioseb, Tampa Bay, FL). ${ }^{38,60,61}$ In these experiments the rats were allowed to roam freely for $5 \mathrm{~min}$. and their movement were captured using an overhead camera (BioEPM3C, EB-Instruments, Tampa Bay, FL). From the captured video, the number of entries into each arm as well as the total time spent in the open vs. closed arms were extracted. Compared with the control rats, the stressed rats avoided the open arm. The rats exposed to the cat showed a decrease in the time spent at the open arm and an increase in the time spent at the closed arm. ${ }^{38,58-61}$ Further, the rats developing stress were identified through a label-free in vivo electron paramagnetic resonance (EPR) test. ${ }^{38}$ The stressed rats showed an elevated level of reactive oxygen species (ROS) in their brains compared to the control rats. ${ }^{38}$

The rats from the control and PTSD groups were humanely euthanized via inhalation of carbon dioxide. Immediately after, transcardial perfusion was performed using $10 \mathrm{mM}$ phosphatebuffered saline (PBS) solution. To fix the tissue, this step was followed by transcardial perfusion with $4 \%$ phosphate-buffered paraformaldehyde (PB-PFA) solution. We immediately harvested the whole brain using cranial dissection. The brains were cryosectioned into $40 \mu \mathrm{m}$ thick slices that were kept in $1 \mathrm{X}$ PBS at $4{ }^{\circ} \mathrm{C}$. The tissue sections containing the paraventricular nucleus of the thalamus (PVT) region were found by using the rat brain stereotaxic coordinates in the Bregma number regions of $-1.20 \mathrm{~mm}$ to $-3.6 \mathrm{~mm}$ (see supplementary Figure S1).

\subsection{Histology}


Hematoxylin and Eosin (H\&E) staining protocol was followed for the histology observations. First, the nuclei were stained with alum hematoxylin. After rinsing, the samples were dipped in acidified alcohol ( $1 \mathrm{ml}$ concentrated $\mathrm{HCl}$ with $400 \mathrm{ml} 70 \%$ ethanol). Afterwards, samples were rinsed again and stained with eosin for 2 min. Finally, they were dehydrated and mounted on standard $75 \mathrm{~mm}$ x $25 \mathrm{~mm}$ microscope slides.

\subsection{Raman Spectroscopy and Microscopy}

The Raman spectra of pure lipids in powder form were obtained with a Renishaw inVia Reflex Raman Spectroscope with a $785 \mathrm{~nm}$ laser with an exposure time of $20 \mathrm{~s}$ and $100 \%$ power. All the Raman spectra from brain samples were obtained under PBS (1X) immersion conditions with an exposure time of $10 \mathrm{~s}$, step size of $20 \mu \mathrm{m}$, objective lens of 50X (long-working distance) and with high confocality. In spectral acquisition mode, from each sample at least ten spectra were obtained for statistical analysis. In the imaging mode, for PTSD and Control samples 798 and 702 spectra were collected, respectively, to generate the Raman images. The machine was calibrated using the silicon peak at $520 \mathrm{~cm}^{-1}$.

\subsection{Data Processing and Statistical Analysis}

All the Raman spectra were baseline corrected by Renishaw's WiRE 4.4 (Windows-based Raman Environment) software. Preprocessing of the data and the subsequent principal component analysis were performed by Origin 2018 (OriginLab, Northampton, MA). The Min-Max normalization approach was applied to the datasets and smoothing was performed by means of the Savitzky-Golay method. Direct classical least squares analysis (DCLS) method was used to generate the mapping data. Image 1.8 software was used for the quantification of Raman mapping based imaging data. Figures S2-S4 demonstrate the steps for quantification of the Raman maps by 
means of pixel intensity and distribution. Accordingly, all the images were first converted into 8bit format. The weighted mean value of brightness (pixel value) as well as the regarding histogram data were then calculated for all the pixels in an ROI by using the "Measure" function under the "Analyze" tab of ImageJ software. This value is chosen as a representative for the relative concentration of lipids (Figures S2 and S3). A color thresholding method was used to determine the distribution of lipids. Based on the inspection of all the images, a brightness value of 111 (in order to have a good contrast) was selected as a threshold in all the images. By keeping the threshold constant, we analyzed the distribution of lipids in each Raman map (Figure S4).

The Raman spectral data were analyzed using principal component analysis (PCA). ${ }^{62-65}$ Multivariate analysis using the "Principal Component Analysis for Spectroscopy" toolbox of Origin 2018 was used to perform the PCA. The spectral differences among the data sets were described by the principal components (PC). Each of the Raman spectra is described as a point on a score plot when selecting two or more PCs. Finally, the clustering of the data on the score plot and their vibrational fingerprint assignment is obtained by the loading plot.

\subsection{MALDI Analysis}

A matrix assisted laser desorption/ionization (MALDI)-TOF/TOF mass spectrometer (UltrafleXtreme, Bruker Daltonics, Billerica, MA, USA) was utilized for mass spectrometry imaging (MSI) in positive ion mode. Brain slices were mounted on an indium tin oxide (ITO) coated slides (UniversityWafer, Inc.). Super-DHB (Millipore-Sigma), 2, 5-dihydroxybenzoic acid (DHB, Sigma-Aldrich), and $\alpha$-cyano-4-hydroxycinnamic acid (Millipore-Sigma) were used as the matrix. ${ }^{66}$ The matrix was prepared by dissolving super-DHB (1:1 ratio) in methanol and water solution containing 0.1 vol\% of trifluoroacetic acid, to a final concentration of $10 \mathrm{mg} / \mathrm{mL}$. Tissue sections were dried in a vacuum chamber for about 7 minutes. Afterwards, matrix was uniformly 
deposited on the tissue by means of pneumatic nebulizer (nitrogen gas pressure $=70 \mathrm{kPa}$; liquid flow rate $=100 \mu \mathrm{L} / \mathrm{min}$ ). Lamp heating was used to dry the matrix on the surface of the tissue. All the MS data were smoothed by B-spline fit. A custom code, 'Decimator.vi' written in LabVIEW VI (National Instruments) was utilized for baseline subtraction, averaging, decimation, and denoising of the obtained data. Bruker FlexAnalysis 3.0 software and MSiReader, ${ }^{67,} 68$ an opensource Matlab package, were employed for data analysis after baseline subtraction. Peak assignments were done by using the available databases on lipidmaps.org. To generate MALDI images, 1944 and 539 spectra were collected from PTSD and Control samples, respectively. MALDI images were analyzed by using the same method used for Raman images, as described above.

\section{Results and Discussion}

\subsection{Raman Spectra for the PTSD and Control Tissue}

Figure 1 shows an overview of this study from the animal model to output data. A predator exposure/psychosocial stress regimen was utilized to develop PTSD in the rat. For verification of PTSD induction, behavioral analysis, neurotransmitter changes, and oxidative stress analysis were performed as described in the previous study. ${ }^{38}$ In order to demonstrate the location of the PVT region in rat brain, Figure 2 shows $\mathrm{H} \& \mathrm{E}$ (Figure 2a, b), bright field (Figure 2c, d) and Raman (Figure 2e, f) images of the control and PTSD brain tissues. Magnified PVT region is shown next to the image of full brain slice. The PVT region is visible with a white color in $\mathrm{H} \& \mathrm{E}$ images and a darker color in bright field images. Raman images (Figure 2e, f) were plotted at the peak position of $1002 \mathrm{~cm}^{-1}$ for control and PTSD samples. This peak is the most abundant peak in most of the biological samples. It is assigned to the aromatic ring C-C stretching mode and usually demonstrates the existence of phenylalanine amino acid. ${ }^{35}$ Accordingly, the PVT region is 
distinguishable from the surrounding area due to different contrast in all the three imaging methods.

Figure $3 \mathrm{a}$ and $3 \mathrm{~b}$ show the magnified view $(12 \mathrm{X})$ of $\mathrm{H} \& \mathrm{E}$ images showing the PVT region and the different neuron cell structures inside and outside of the PVT. Accordingly, no morphological change was observed in the aforementioned region. To probe the biochemical changes, Raman experiments were performed on the same region. Raman spectra of control (Figure 3c) and PTSD (Figure 3d) samples illustrate the biochemical changes in the brain in the PVT region due to the applied stress. The diagrams are achieved by plotting 30 different spectra inside the dotted area for each sample. The average value is highlighted by darker colors for both datasets. Raman bands of the brain spectra are assigned to cholesterol $\left(608 \mathrm{~cm}^{-1}\right)$, methionine $(700$ $\left.\mathrm{cm}^{-1}\right)$, DNA/RNA $\left(847 \mathrm{~cm}^{-1}\right)$, phenylalanine $\left(1002 \mathrm{~cm}^{-1}\right)$, acyl chains $\left(\mathrm{C}-\mathrm{C}\right.$ stretch; $\left.1064 \mathrm{~cm}^{-1}\right)$, proteins (C-N stretch; $\left.1127 \mathrm{~cm}^{-1}\right)$, lipids $\left(\mathrm{CH}_{2}\right.$ twist; $\left.1300 \mathrm{~cm}^{-1}\right)$, lipids $\left(\mathrm{CH}_{2}\right.$ bend; $\left.1439 \mathrm{~cm}^{-1}\right){ }^{27}$ The Raman peak positions did not change significantly in the PTSD brain samples compared to control samples (the assignment of all the Raman peaks are in Table S1). However, we found a consistent modulation of Raman intensities between control and PTSD samples. Therefore, we utilized principal component analysis (PCA) method to reveal any possible biochemical changes in the PTSD brain samples. Significant changes were identified by the PCA and scatter plot that were able to discriminate both sample groups, PTSD and control.

\subsection{Raman Fingerprint Spectra Discriminates PTSD and Control}

Figure $3 \mathrm{e}$ shows the score plot from the first two principle components based on 30 spectra chosen from PTSD (pink) and control (blue) tissue samples. The score plot demonstrates a clear segregation and distinct clustering of data obtained from different sample groups. Figure S5 also shows the 3D view of the PCA analysis including the scatter plots from these first three PCs and 
it can distinguish the two sample groups as well. Figures $3 \mathrm{f}-3 \mathrm{~h}$ show the box plot of the distribution of spectral data for the two groups. The spectral data of the PTSD samples were significantly shifted $(P<0.001)$ to the negative PC 1 range (median $=-0.15)$ compared to control (median $=$ 0.18; Figure 3f). Likewise, substantial changes $(P<0.05)$ were observed in the PC 2 (Figure $3 g$ ) data distribution by shifting the median value from -0.14 in the control to 0.03 in the PTSD. However, the changes in PC 3 (Figure 3h) were not significant $(P>0.05)$.

To find the biochemical components in each spectral variation, loading plots (Figures 3i, $3 \mathrm{j}, 3 \mathrm{k})$ of the spectra obtained from the PVT region from PTSD and control are presented. The loading plots demonstrate the major spectral differences of the PCs. The overall contribution from the first three PCs is $\sim 97.6 \%$. Characteristic peaks of each dataset are provided for PC 1 , PC 2 and PC 3 (Figures 3i, 3j, 3k). These characteristic peaks indicate the differences in the various Raman datasets. The loading plot for PC 1 (Figure 3i) was obtained from PTSD and control samples and represents $90.5 \%$ of the spectral variation includes the Raman peaks from all biomolecules types (lipids, proteins, RNA and DNA). For example, 700 (methionine C-S trans), 1002 (phenylalanine C-C aromatic ring stretch), 1085 (phosphodiester groups in nucleic acids), 1300 (lipid $\mathrm{CH}_{2}$ twist) and $1438 \mathrm{~cm}^{-1}$ (lipid $\mathrm{CH}_{2}$ bend) are the prominent peaks for PC $1 ; 849$ (amino acid stretch), 1002, 1300 and 1447 (proteins and lipids $\mathrm{CH}_{2}$ bending) $\mathrm{cm}^{-1}$ are the most distinctive bands for PC 2 (Figure 3j). Moreover, the third compound of PC (Figure 3k) which contains only $0.6 \%$ of the spectral variation, can differentiate between proteins $\left(1002 \mathrm{~cm}^{-1}\right.$, negative value) and lipids ( $1300 \mathrm{~cm}^{-1}$, positive value). As can be seen, some of the most significant distinctive characteristics were observed in the lipid bands. As a result, a considerable change in the concentration of lipids can be derived from the Raman spectra of PTSD brain compared to the control sample. The in-depth discussion of lipid alterations is provided in the following. 


\subsection{Raman Spectroscopic Comparison of Lipids Relevant to Brain Tissue}

In order to track the lipid concentration changes in the rat brain due to acute stress exposure, Raman spectra of the eleven most common lipids in brain ${ }^{32,69-71}$ were acquired by collecting the Raman signal from pure reference lipid samples by means of the $785 \mathrm{~nm}$ excitation laser. As can be seen in Figures $4 a-4 e$, different lipids have different Raman spectra which distinguish them from the other lipids. The spectral region from $400-1800 \mathrm{~cm}^{-1}$ shows the fingerprint region for each lipid. The peaks in the wavenumber region $2700-3500 \mathrm{~cm}^{-1}$ are due to the $\mathrm{CH}$, and $\mathrm{OH}$ stretch (Figure S6). For instance, peaks from $2845-2868 \mathrm{~cm}^{-1}$ are due to the $=\mathrm{CH}_{2}$ symmetric stretch, $2870-2904 \mathrm{~cm}^{-1}$ are from the $=\mathrm{CH}_{2}$ asymmetric stretch, peaks from $2905-2940 \mathrm{~cm}^{-1}$ are due to the $-\mathrm{CH}_{3}$ symmetric stretch, $2941-2970 \mathrm{~cm}^{-1}$ are from the $-\mathrm{CH}_{3}$ asymmetric stretch, and the Raman peaks from $3000-3015 \mathrm{~cm}^{-1}$ are due to the unsaturated $=\mathrm{CH}$ bond stretch. ${ }^{27}$ Figure $4 \mathrm{a}$ shows the comparison of cholesterol (FC) and cholesteryl palmitate (or cholesterol ester, $\mathrm{CE}$ ) (the molecular structure is in Figure S7). The Raman band due to ester group at $1737 \mathrm{~cm}^{-1}$ is absent in cholesterol. Spectral band at 1065,1130 , and $1297 \mathrm{~cm}^{-1}$ are due to palmitic acid group in the cholesterol ester. ${ }^{69}$ The intense band at $1440 \mathrm{~cm}^{-1}$ is due to acyl group $\left(\mathrm{CH}_{2}\right.$ or $\mathrm{CH}_{3}$ scissor $)$. Further, the following band shift was observed from cholesterol to cholesterol ester: 422 to 428 , 548 to 538 ( $\mathrm{CH}_{2}$ bend), 608 to 614 (ester group), and 1672 to $1667 \mathrm{~cm}^{-1}$ (C=C stretch).

Next, Figure $4 \mathrm{~b}$ compares glyceryl palmitate (or triacylglyceride, TAG) and phosphatidic acid (PA) (Figure S8). They share quite similar structures with one palmitic acid chain in TAG replaced by a phosphate group for PA. The band due to ester group appears at $1737 \mathrm{~cm}^{-1}$ for PA. The ester band for TAG splits into two at 1728 and $1743 \mathrm{~cm}^{-1}$ indicating that they are in a different surrounding environment compared to PA. The three small bands at 587, 607, and $630 \mathrm{~cm}^{-1}$ are due to glycerol. ${ }^{72}$ The band due to stretching of P-O vibration of $\mathrm{PO}_{4}$ group is at $993 \mathrm{~cm}^{-1}{ }^{73}$ The 
intense bands at 1061 (C-C stretch), 1130 (C-C stretch), $1297\left(\mathrm{CH}_{2}\right.$ twist), and $1444 \mathrm{~cm}^{-1}$ $\left(\mathrm{CH}_{2} / \mathrm{CH}_{3}\right.$ scissoring) are due to fatty acid chains. Figure $4 \mathrm{c}, \mathrm{d}$ show the comparison of different phospholipids. As shown in Figure S9, the parent group of phospholipids (PE, PI, PC, PS) is the phosphatidic acid (PA). Therefore, the Raman bands of phospholipids share features similar to that of phosphatidic acid. The band for phosphatidylethanolamine (PE) at $758 \mathrm{~cm}^{-1}$ is assigned to ethanolamine (Figure 4c, Figure S10). Similarly, the band at $1095 \mathrm{~cm}^{-1}$ of the PE Raman spectrum is due to phosphodioxy groups $\mathrm{PO}_{2}^{-}$(P-O stretch). At low wavenumbers between $200-1000 \mathrm{~cm}^{-1}$, distinct differences among phospholipids were observed (Figure 4d). Phosphatidylinositol (PI) shows peaks at $234,419,523,721,770,847,873,888$, and $974 \mathrm{~cm}^{-1}$; Phosphatidylserine (PS) shows peaks at $234,524,608,753,810,848,873,888,927$, and $974 \mathrm{~cm}^{-1}$. The PI and PS have bands at $1658 \mathrm{~cm}^{-1}(\mathrm{C}=\mathrm{C}$ stretch) which are absent in PA and PE. All the phospholipids have band for ester $\left(1739 \mathrm{~cm}^{-1}\right)$ and acyl chains $\left(1065,1130,1298,1437 \mathrm{~cm}^{-1}\right.$; Figure S10).

Figure 4e compares the Raman spectra obtained from sphingolipids (see Figure S11 for the molecular structures). Sphingolipids are composed of lipids, a ceramide backbone, and glucose rings with or without linker groups; the linker group for galactocerebroside (GalCer) is galactose, sulfate containing monosaccharide for sulfatide (ST), and oligosaccharides for ganglioside (GM). As seen in Figure S11 and S12 (also Figure 4e), Raman signature of lipids (at 1064, 1129, 1298, $\left.1437 \mathrm{~cm}^{-1}\right)$, ceramide backbone $\left(1657,1671 \mathrm{~cm}^{-1} ; \mathrm{C}=\mathrm{C}\right.$ stretch$)$, and sugar chain $\left(1370 \mathrm{~cm}^{-1}\right.$; Figure S12) is clearly present. Figure S12 also shows that the area under the peak at $1370 \mathrm{~cm}^{-1}$ is the highest for ganglioside (GM) and lowest for sphingomyelin (SM). This agrees well with the molecular structure of GM, which has the maximum saccharide content (Figure S11) and that of SM, which has no glucose group. Further, we observed that the peak ratio of 1370 (sugar chain) to $1297 \mathrm{~cm}^{-1}$ (fatty acid) is the highest for GM. The Raman peak at $890 \mathrm{~cm}^{-1}$ (C-O-O skeletal 
mode) is present in all the sphingolipids except SM as SM does not have any C-O-O group (Figure S13). The sulfate band in ST is characterized by 614 and $995 \mathrm{~cm}^{-1}$ (Figure S14). ${ }^{74}$ Finally, sphingomyelin is composed of a phosphatidylcholine (PC) residue, which is characterized by Raman peaks at 718 and $875 \mathrm{~cm}^{-1}$ (Figure 4e, S15). Another key difference between the backbone of phospholipids and ceramide structure is the amide bond $\left(1669 \mathrm{~cm}^{-1}\right.$; Figure $\left.4 \mathrm{e}, \mathrm{S} 15\right)$ in the ceramide backbone instead of the ester band $\left(1739 \mathrm{~cm}^{-1}\right)$ of phospholipids.

Further, we utilized one-way multivariate statistical analysis coupled with the PCA approach to separate different populations of lipids. PCA analysis was performed on the 11 most common lipids found in brain tissue. Figure $4 \mathrm{f}$ represents the PCA scatterplot results obtained from the first-derivative of the Raman data in various data sets of lipid spectra. The $95 \%$ confidence ellipse of the clustered group PCA distribution is also shown in the plots. The PCA analysis of the raw data did not cluster different classes of lipids separately and overlap among classes can be seen (Figure S16a). However, after normalization, smoothing, and taking the $1^{\text {st }}$ derivative of the data, considerable separation among different lipid clusters was observed in the PCA results (Figure S16b-d). Meanwhile, the second-derivative did not further improve separation of lipid clusters. Therefore, it can be concluded that the first-derivative provides the best PCA results for our data set (Figure 4f).

To include more variance among the data sets, PC 3 of the first-derivative was also calculated to plot the 3D PCA result (Figure S16e). Figures 4g-4i show the PC values of firstderivative for different lipids. Each bar shows the mean value of the data. By performing ANOVA on the PC 1 data set, it can be seen that the PC 1 value for every lipid is significantly different $(P<$ 0.001) from the other lipids (see Table S2). Similarly, the same significant difference $(P<0.001)$ was also observed for PC 2 data set except for phosphatidylserine vs. sphingomyelin, 
phosphatidylserine vs. ganglioside, and ganglioside vs. sphingomyelin (see Table S2). Furthermore, the PC 3 data also showed significant differences $(P<0.001)$ for most of the lipids (see Table S2). The corresponding loading plots of PC 1, PC 2, and PC 3 show the major peaks responsible for the clustering of the data set (Figure $4 \mathrm{j}, 4 \mathrm{k}, 41$ ). The percentage variance described by PC 1, PC 2, and PC 3 was 36.4\%, 21.3\%, and 9.8\%, respectively. Analysis of the loading plots showed that the prominent Raman peaks of the lipids show the most variance at 704 (characteristic band for cholesterol), 1133 (acyl chain, palmitic and fatty acids), 1300 (lipid $\mathrm{CH}_{2}$ twist mode) and 1293 (methylene) $\mathrm{cm}^{-1}$ peak positions. As a result, it can be concluded that Raman spectra of the most abundant lipids in the brain are statistically different from each other. Therefore, we can use statistical methods to compare the lipid concentration in control and PTSD samples.

\subsection{Raman Identifies Differences in Lipid Signature in PTSD and Control Brain Tissues}

In order to compare the amount of lipid distribution in control and PTSD samples, we used direct classical least squares analysis (DCLS) method. ${ }^{75-77}$ According to this model, the mixture spectrum can be modeled as a linear mixture of various reference spectra. The DCLS model is useful when reference spectra are available from pure samples. Accordingly, we first acquired the Raman signal from the PVT region of both control and PTSD specimens. Afterwards, the Raman spectra of pure lipids (Figure 4a-e) were used to quantify each lipid in terms of relative concentration and distribution. Figures S17, S18 and S19 illustrate the Raman maps of eleven commonly found lipids in the PVT region of the rat brain (the bars beside each image indicate the value of the correlation coefficient between the corresponding lipid and brain spectra). After plotting the respective maps for each lipid, ImageJ software was used to analyze the relative concentration and distribution of lipids. To this end, the intensity value of brightness in each pixel and also distribution of pixels is needed. The intensity values give the relative concentration 
change of each lipid. The distribution of pixel intensity describes the distribution of lipids in the PVT region. For a tangible example, comparative Raman maps of cholesterol, galactocerebroside, sulfatide and ganglioside are demonstrated in Figure 5a-5f. As can be seen, the relative concentration of cholesterol and sulfatide is greater in the PTSD sample compared to the control. In order to quantify the relative concentration values, we used pixel value which is a number between $0-255$. The value 0 indicates the minimum brightness and 255 indicates the maximum brightness of a pixel. These values were obtained from 8-bit images by means of ImageJ software. Figure S20 shows the pixel value distributions of different lipids in control and PTSD samples. The relative shifts of the peaks to the right (higher pixel values), indicates an increase in the relative concentration of lipids. Therefore, we can acquire the weighted mean value for each lipid from this diagram that directly indicates the concentration changes of different lipids.

Figure 5g demonstrates the acquired weighted mean values for different lipids in PTSD and control samples. Accordingly, the relative concentration of phosphatidylinositol, phosphatidylserine, phosphatidylethanolamine, cholesteryl palmitate, cholesterol, sphingomyelin and sulfatide experienced an increase by $23 \%, 44.6 \%, 20.6 \%, 10.2 \%, 35 \%, 32.2 \%, 54 \%$ in PTSD sample, respectively. On the other hand, relative concentration of glyceryl tripalmitate and ganglioside decreased by $47.1 \%$ and $23.3 \%$, respectively, relative to control tissue. Meanwhile, galactocerebroside and phosphatidic acid did not change noticeably. Stress response is followed by the release of corticosterone. ${ }^{57}$ This hormone, which is derived from cholesterol, mediates the pathologic responses of severe stress by binding to intercellular receptors. It is believed that the concentration of neutral lipids (cholesteryl palmitate and cholesterol) in brain is correlated with the level of corticosterone. ${ }^{1}$ Therefore, higher concentration of cholesterol indicates the higher level of corticosterone in brain. Accordingly, the changes in lipid concentration have numerous 
impacts on physiological mechanisms of stress-related disorders. Changes in the concentration of phospholipids (phosphatidylinositol, phosphatidylserine and phosphatidylethanolamine) and sphingolipids (sphingomyelin, ganglioside, glyceryl tripalmitate and sulfatide) also reveal the alteration of phospholipase A2 (PLA2) which is associated with inflammatory processes in brain. $.77,78$

Apart from the relative concentration of lipids, we have also analyzed the areal distribution of lipids in PVT region. The areal distribution is given as the percentage of occupied areal by each lipid in PVT region (Figure 5h). By comparing the results from Figures $5 \mathrm{~g}$ and $5 \mathrm{~h}$, it can be observed that for some lipids (e.g. cholesterol and ganglioside), the distribution percentage of lipid does not follow the trend of relative concentration. For example, for cholesterol, the lipid areal distribution decreases slightly despite the increase in relative concentration of the lipid. Similarly, for ganglioside the distribution percentage is almost constant while the relative concentration is lower. The reason for the differences is due to the fact that we are not taking into account the pixel intensity values while calculating the areal distribution percentage. In other words, we are using a binary logic for distribution percentage to choose whether a pixel is ON (particular lipid is present) or OFF (lipid is absent). Therefore, relative concentration and areal distribution should be utilized side by side in order to achieve a fair judgment of lipidome alterations in the brain tissues.

\subsection{MALDI-MS Imaging of Brain Tissue}

To validate the Raman imaging results, we utilized MALDI mass spectrometry imaging (MALDI-MSI) as a conventional lipidomic studies method to elucidate the role of lipids in PTSD. The obtained lipidome profile is affected by the choice of ionization mode. ${ }^{79-84}$ Since wide range of lipids can be ionized in positive mode, its widely used for lipidomics.$^{79}$ However, phospholipids

such as PI, PS, and PA yield better results in negative ionization mode. ${ }^{85}$ Although we used both 
modes, only the MALDI images obtained in positive mode are shown here. The MALDI-MS spectra obtained in the positive ion mode from the PVT region of the control and PTSD brain tissue sample are shown in Figure 6a. The comparison of positive and negative ion mode spectra is shown in the Supporting Information Figure S21. The MS spectra display both low molecular weight (400 - 650 Da) and high molecular weight (700 - $1200 \mathrm{Da})$ lipid species. Positive ion mode also allowed observation of low molecular weight lipids in the control brain tissue such as lysophosphatidylcholines (LPC O-15:1, $\mathrm{m} / \mathrm{z}$ 466.3), which are generally masked by the signal from the matrix. ${ }^{86}$ Intense signal at $m / z 663.4$ for the control brain sample corresponds to PA $30: 3 ; \mathrm{O} 3$. Other observed species were phosphatidylglycerols (PG 28:2, $\mathrm{m} / \mathrm{z}$ 663.4). For the PTSD sample, the intense mass peaks correspond to fatty acyls such as N-acyl taurines (NAT 20:4;O, m/z 466.2), glycerophospholipids (PA 39:8, PG 33:3, PA 37:5, PG 31:0, PA O-38:5, PG O-31:1, PG 30:1, m/z 731.5; PC O-42:5, PC 41:5, PE 44:5, PC O-40:2, $\mathrm{m} / \mathrm{z}$ 850.7), glycerolipids such as di(acyl|alkyl)glycerols (DG 42:8, $\mathrm{m} / \mathrm{z}$ 731.5; DG 44:3, $\mathrm{m} / \mathrm{z}$ 753.6), tri(acyl|alkyl)glycerols (TAG 43:3, $m / z$ 753.6), and sphingolipids such as hexosyl ceramides (HexCer 42:1;O3, $\mathrm{m} / \mathrm{z}$ 850.7).

Figure $6 \mathrm{~b}$ shows the bright field optical image of the MALDI-MS sample. The distribution of each lipid is shown in Figure 6c and 6e (also see Figure S22). We are able to observe the pathological lipid changes in the PVT region of the brain due to PTSD. For example, the positive ion image of sulfatides (ST 38:1, m/z 890.5; mFigure 6c) showed an accumulation of sulfatides near the PVT of the PTSD rat. A similar elevated level of ST after traumatic brain injury was observed in an earlier study. ${ }^{87}$ Figure $6 \mathrm{~d}$ compares the Raman and MALDI image analysis results. Figure 6d shows the ratio of PTSD to control calculated from the Raman images using the mean value of pixel intensity (pink bars) for different lipids. The corresponding PTSD to control ratio calculated from each MALDI-MS image (blue bars) is also compared with the Raman ratio. The 
ratio, $R=1$ indicates no change, $R>1$ indicates increase, and $R<1$ indicates decrease of specific lipids in PTSD samples compared to the control samples. Accordingly, an increase in the relative concentration value of phosphatidylinositol (PI), phosphatidylserine (PS), phosphatidylethanolamine (PE), cholesterol (FC), sphingomyelin (SM) and sulfatide (ST) is observed by both Raman and MALDI imaging methods in the PTSD sample compared to the control. Moreover, the decrease in the ganglioside (GM) and glyceryl tripalmitate (TAG) in the PTSD sample was also confirmed by the MALDI images. No significant change was observed for galactocerebroside (GalCer) and phosphatidic acid (PA) by any of the methods. Only cholesteryl palmitate and glyceryl tripalmitate displayed a difference in its relative concentration between MALDI detection and Raman detection. Therefore, the majority of the detected signals in MALDI are in agreement with the Raman imaging analysis.

Figure 6e shows representative MALDI-MS images of selected lipid species in control and PTSD brain samples. The images were constructed using the following mass peaks: phosphatidylserine (PS 34:2, $\mathrm{m} / \mathrm{z}$ 760.5; PS 39:0, $\mathrm{m} / \mathrm{z}$ 834.6; PS 39:7, $\mathrm{m} / \mathrm{z}$ 842.5; PS 40:1, $\mathrm{m} / \mathrm{z}$ 868.6; PS 43:4, $m / z$ 882.6), phosphatidylinositol (PI 31:3, $\mathrm{m} / z$ 829.4; PI O-35:2, $\mathrm{m} / z$ 857.6; PI O37:3, $m / z$ 883.6; PI O-37:2, m/z 885.6), sphingomyelin (SM 34:2-O2, $m / z$ 723.5; SM 33:0-O2, $m / z$ 729.5; SM 34:1-O2, m/z 741.5; SM 36:2-O2, m/z 767.5), phosphatidic acid (PA O-31:0, $m / z$ 643.5; PA O-33:1 \& PA 32:1, $\mathrm{m} / \mathrm{z}$ 647.5; PA O-35:3, $\mathrm{m} / \mathrm{z}$ 671.5; PA 35:5, $\mathrm{m} / \mathrm{z}$ 719.4), cholesterol (FC 24:5, $m / z \quad 401.2 ;$ FC 27:1-O, $m / z \quad 409.3 ;$ FC 26:2-O3, $m / z \quad 425.3$; ST 27:1-O, $m / z \quad 425.3$ ), galactocerebroside (GalCer 34:1-O2, m/z 722.5; GalCer 40:2-O2, m/z 804.6; GalCer 40:1-O2, $m / z$ 806.6; GalCer 41:2-O2, $m / z$ 834.6), triacylglyceride (TAG 50:9, $\mathrm{m} / \mathrm{z}$ 817.6; TAG 52:9, $\mathrm{m} / \mathrm{z}$ 845.7; TAG 50:2, $\mathrm{m} / \mathrm{z}$ 869.7; TAG 52:3, $\mathrm{m} / \mathrm{z}$ 895.7; TAG 58:13, $\mathrm{m} / \mathrm{z}$ 921.7), cholesterol ester (CE 18:3, $\mathrm{m} / \mathrm{z}$ 669.6; CE 20:5 \& CE 18:2, m/z 671.6; CE 22:0, $\mathrm{m} / \mathrm{z}$ 731.7; CE 24:1, $\mathrm{m} / \mathrm{z}$ 757.7), 
ganglioside (GM or Hex(3)-HexNAc-KDN-Cer 36:1-O2, m/z 1543.8; GM 36:1-O2, m/z 1562.9; GM or Hex(4)-HexNAc-Fuc-Cer 36:1-O2, m/z 1563.9), phosphatidylethanolamine (PE 36:6, m/z 736.5; PE O-36:6, $\mathrm{m} / \mathrm{z}$ 744.5; PE O-37:1, $\mathrm{m} / \mathrm{z}$ 746.6; PE 37:6, $\mathrm{m} / \mathrm{z}$ 750.5; PE 38:7, $\mathrm{m} / \mathrm{z}$ 762.5; PE O-36:3, $\mathrm{m} / \mathrm{z}$ 766.5; PE O-37:1, $\mathrm{m} / \mathrm{z}$ 768.6; PE 40:7, $\mathrm{m} / \mathrm{z}$ 790.5; PE 39:0, $\mathrm{m} / \mathrm{z}$ 790.6; PE 40:6, $m / z$ 792.6; PE 37:3, m/z 794.5; PE O-39:2, m/z 794.6).

The membrane lipid PI plays an important role in the signal transduction. ${ }^{88}$ Although experiments performed in a different region of the brain (prefrontal cortex, PFC, and hippocampus), Oliveira et al. also reported an increase of PI in the rat brain due to chronic stress. ${ }^{32}$ The increase of other phospholipids such as PE and PS reported in our study are supported by other literature observations using neurodegenerative disease models. ${ }^{89-91}$ In addition to cholesterol and phospholipids, sphingolipids are the most common membrane lipids in the brain. ${ }^{88,92,93}$ Sphingolipids such as gangliosides are implicated in brain development, memory formation as well as synaptic transmission. ${ }^{94}$ Our results show a decrease in the gangliosides concentration in PTSD samples, which is supported by the observations from Martin et al. and Kracun et al. using the brain tissue of human subjects. ${ }^{95}{ }^{96}$ Further study by Oliveira et al. showed that the alteration in lipid levels in the brain is area dependent. They observed an increase of the PI in the hippocampus and a decrease in PE in the PFC, but no changes in the phospholipid levels in the amygdala or cerebellum. ${ }^{97}$ These findings suggest that lipidome analysis should be performed in specific areas of the brain for meaningful comparisons. Although the lipid distribution in the brain is dynamic and complex, it is suggested to play some role in depression and anxiety disorders. ${ }^{3}$ The knowledge gained from this study may provide lipid-based targets for disease prevention and treatment. 


\section{Conclusion}

In this study, we have evaluated the application of Raman spectroscopy and imaging for the quantitative analysis of lipid concentrations in paraventricular thalamic nucleus (PVT) of posttraumatic stress disorder (PTSD), and control brain tissue. Raman spectroscopy provided a new tool to non-invasively monitor the lipid changes in the brain tissue. Combining with histology and MALDI mass spectrometry imaging, we have performed a parallel study with Raman imaging and multivariate data analysis to validate the distinguishing of different lipids in brain tissue. Further, we used a direct classical least squares analysis approach for rendering Raman maps and imaging the lipid concentration in the PVT region. By means of this technique and various image processing methods, we have demonstrated the relative alteration of lipids in PVT region before and after inducing PTSD. Our results show a relative increase in the concentration of phosphatidylinositol (28\%), phosphatidylserine (43\%), phosphatidylethanolamine $(11 \%)$, cholesteryl palmitate $(4 \%)$, cholesterol (27\%), sphingomyelin (37\%) and sulfatide (58\%) in the PTSD sample. Meanwhile, a relative decrease in the concentration of glyceryl tripalmitate $(71 \%)$ and ganglioside (32\%) was observed. However, the relative concentration of galactocerebroside and phosphatidic acid did not change noticeably in the PTSD samples compared to the control. The higher relative concentration of cholesterol and cholesteryl palmitate are directly related to the level of corticosterone.

Changes in the concentration of phospholipids and sphingolipids are associated with inflammatory processes in the brain by changing the level of phospholipase A2 (PLA2). Similar Raman imaging methods can be applied to other regions of brain and other types of brain disorders. The Raman scattering-based label-free method could open new way to perform lipidomic studies on cells and tissue with high spatial resolution for fast and non-destructive analysis. 


\section{Supporting Information}

Paraventricular nucleus of the thalamus (PVT) region inside rat brain; The method used for quantification of Raman maps by means of pixel values; Quantification of phosphatidylinositol base on pixel value method for control and PTSD samples; Calculating the areal distribution percentage base on thresholding method; 3D view of PCA analysis; High-frequency region of Raman spectra for different lipids; Comparison of cholesterol (FC) and cholesteryl palmitate (or cholesterol ester, CE) chemical structures; Comparison of glyceryl palmitate (or triacylglyceride, TAG) and phosphatidic acid (PA) chemical structures; Chemical structure of different phospholipids (PE, PI, PC, PS); Comparison of the Raman spectra obtained from sphingolipids; Raman spectrum of sphingomyelin; PCA analysis of the most common lipids in brain tissue for raw data, normalized data, smoothed data and $2^{\text {nd }}$ derivative data; Raman maps of phosphatidylserine, phosphatidylinositol, phosphatidylethanolamine, cholesterol, galactocerebroside, glyceryl tripalmitate, phosphatidic acid, sphingomyelin, sulfatide, ganglioside and cholesteryl palmitate in PVT region of the rat brain; Pixel value distributions of different lipids; Comparison of positive and negative ion mode spectra; MALDI images acquired from PVT region; Peak positions of Raman spectrum for control and PTSD samples; ANOVA with Levene test for Homoscedasticity for the Raman spectra of the lipids

\section{Acknowledgements}

Raman experiments were performed at LSU's Shared Instrumentation Facility (SIF). Mass spectrometry experiments were performed at the LSU Mass Spectrometry Facility (MSF). MRG acknowledges LSU start-up fund and Louisiana Board of Regents Support Fund (RCS Award Contract Number: LEQSF(2017-20)-RD-A-04). AC is supported by LSU Economic Development Assistantships (EDA). Authors thank Dr. Olalekan Ogundele for sample preparation of rat brains. 


\section{References}

1. D. Danan and H. Cohen, European Neuropsychopharmacology, 2018, 28, S36.

2. D. Piomelli, G. Astarita and R. Rapaka, Nature Reviews Neuroscience, 2007, 8, 743-754.

3. C. P. Müller, M. Reichel, C. Mühle, C. Rhein, E. Gulbins and J. Kornhuber, Biochimica et Biophysica Acta (BBA) - Molecular and Cell Biology of Lipids, 2015, 1851, 1052-1065.

4. J. K. Prasain, L. Wilson, H. D. Hoang, R. Moore and M. A. Miller, Metabolites, 2015, 5, 677-696.

5. R. B. Chan, T. G. Oliveira, E. P. Cortes, L. S. Honig, K. E. Duff, S. A. Small, M. R. Wenk, G. Shui and G. Di Paolo, Journal of Biological Chemistry, 2012, 287, 2678-2688.

6. A. Naudí, R. Cabré, M. Jové, V. Ayala, H. Gonzalo, M. Portero-Otín, I. Ferrer and R. Pamplona, in International review of neurobiology, Elsevier, 2015, vol. 122, pp. 133-189.

7. Y. Tajima, M. Ishikawa, K. Maekawa, M. Murayama, Y. Senoo, T. Nishimaki-Mogami, H. Nakanishi, K. Ikeda, M. Arita and R. Taguchi, Lipids in health and disease, 2013, 12, 68.

8. D. Cheng, A. M. Jenner, G. Shui, W. F. Cheong, T. W. Mitchell, J. R. Nealon, W. S. Kim, H. McCann, M. R. Wenk and G. M. Halliday, PloS one, 2011, 6.

9. V. Ruipérez, F. Darios and B. Davletov, Progress in lipid research, 2010, 49, 420-428.

10. P. L. Wood, M. D. Filiou, D. M. Otte, A. Zimmer and C. W. Turck, Schizophrenia research, 2014, 159, 365-369.

11. M. Orešič, T. Seppänen-Laakso, D. Sun, J. Tang, S. Therman, R. Viehman, U. Mustonen, T. G. Van Erp, T. Hyötyläinen and P. Thompson, Genome medicine, 2012, 4, 1.

12. J. A. Hamilton, C. J. Hillard, A. A. Spector and P. A. Watkins, Journal of Molecular Neuroscience, 2007, 33, 2-11.

13. E. H. Seeley and R. M. Caprioli, Trends in biotechnology, 2011, 29, 136-143.

14. B. Brügger, Annual review of biochemistry, 2014, 83, 79-98.

15. T. C. Rohner, D. Staab and M. Stoeckli, Mechanisms of ageing and development, 2005, 126, 177-185.

16. J. Soltwisch, H. Kettling, S. Vens-Cappell, M. Wiegelmann, J. Müthing and K. Dreisewerd, Science, 2015, 348, 211-215.

17. M. Jermyn, K. Mok, J. Mercier, J. Desroches, J. Pichette, K. Saint-Arnaud, L. Bernstein, M.-C. Guiot, K. Petrecca and F. Leblond, Science translational medicine, 2015, 7, 274ra219-274ra219.

18. J. Desroches, M. Jermyn, K. Mok, C. Lemieux-Leduc, J. Mercier, K. St-Arnaud, K. Urmey, M.-C. Guiot, E. Marple and K. Petrecca, Biomedical optics express, 2015, 6, 2380-2397.

19. J. M. Surmacki, L. Ansel-Bollepalli, F. Pischiutta, E. R. Zanier, A. Ercole and S. E. Bohndiek, Analyst, 2017, 142, 132-139.

20. A. C. S. Talari, Z. Movasaghi, S. Rehman and I. U. Rehman, Applied spectroscopy reviews, 2015, 50, 46-111.

21. S. Ohira, H. Tanaka, Y. Harada, T. Minamikawa, Y. Kumamoto, S. Matoba, H. Yaku and T. Takamatsu, Scientific reports, 2017, 7, 42401.

22. H. Yang, C. Zhao, R. Li, C. Shen, X. Cai, L. Sun, C. Luo and Y. Yin, Analyst, 2018, 143, 2235-2242.

23. E. Timchenko, P. Timchenko, L. Volova, S. Pershutkina and P. Y. Shalkovskaya, Journal of Optical Technology, 2018, 85, 73-76. 
24. M. Haifler, I. Pence, Y. Sun, A. Kutikov, R. G. Uzzo, A. Mahadevan-Jansen and C. A. Patil, Journal of biophotonics, 2018, 11, e201700188.

25. C. J. Saatkamp, M. L. de Almeida, J. A. M. Bispo, A. L. B. Pinheiro, A. B. Fernandes and L. Silveira, Journal of biomedical optics, 2016, 21, 037001.

26. E. E. de Sousa Vieira, J. A. M. Bispo, A. B. Fernandes and L. Silveira, 2016.

27. K. Czamara, K. Majzner, M. Z. Pacia, K. Kochan, A. Kaczor and M. Baranska, Journal of Raman Spectroscopy, 2015, 46, 4-20.

28. H. Wu, J. V. Volponi, A. E. Oliver, A. N. Parikh, B. A. Simmons and S. Singh, Proceedings of the National Academy of Sciences, 2011, 108, 3809-3814.

29. S. Huang, R. Pandey, I. Barman, J. Kong and M. Dresselhaus, ACS Photonics, 2018, 5, 2978-2982.

30. R. Tuma, Journal of Raman Spectroscopy, 2005, 36, 307-319.

31. A. Rygula, K. Majzner, K. M. Marzec, A. Kaczor, M. Pilarczyk and M. Baranska, Journal of Raman Spectroscopy, 2013, 44, 1061-1076.

32. T. G. Oliveira, R. B. Chan, F. V. Bravo, A. Miranda, R. R. Silva, B. Zhou, F. Marques, V. Pinto, J. J. Cerqueira and G. Di Paolo, Molecular psychiatry, 2016, 21, 80.

33. K. Kochan, E. Maslak, C. Krafft, R. Kostogrys, S. Chlopicki and M. Baranska, Journal of biophotonics, 2015, 8, 597-609.

34. I. s. P. Santos, P. J. Caspers, T. C. Bakker Schut, R. van Doorn, V. Noordhoek Hegt, S. Koljenović and G. J. Puppels, Analytical chemistry, 2016, 88, 7683-7688.

35. Z. Movasaghi, S. Rehman and I. U. Rehman, Applied Spectroscopy Reviews, 2007, 42, 493-541.

36. M. J. Bovin, B. P. Marx, F. W. Weathers, M. W. Gallagher, P. Rodriguez, P. P. Schnurr and T. M. Keane, Psychological Assessment, 2016, 28, 1379.

37. A. De Jongh, P. A. Resick, L. A. Zoellner, A. Van Minnen, C. W. Lee, C. M. Monson, E. B. Foa, K. Wheeler, E. t. Broeke and N. Feeny, Depression and Anxiety, 2016, 33, 359369.

38. P. J. Ebenezer, C. B. Wilson, L. D. Wilson, A. R. Nair and J. Francis, PloS one, 2016, 11, e0160923.

39. G. Ronzoni, A. del Arco, F. Mora and G. Segovia, Psychoneuroendocrinology, 2016, 70, $1-9$.

40. S. Seetharaman, M. Fleshner, C. R. Park and D. M. Diamond, Brain and behavior, 2016, 6, e00458.

41. J. Deslauriers, M. Toth, A. Der-Avakian and V. B. Risbrough, Biological psychiatry, 2018, 83, 895-907.

42. L. M. Shin, S. L. Rauch and R. K. Pitman, Annals of the New York Academy of Sciences, 2006, 1071, 67-79.

43. H. Manjoch, E. Vainer, M. Matar, G. Ifergane, J. Zohar, Z. Kaplan and H. Cohen, Behavioural brain research, 2016, 306, 91-105.

44. Y. Levkovitz, D. Fenchel, Z. Kaplan, J. Zohar and H. Cohen, European Neuropsychopharmacology, 2015, 25, 124-132.

45. S. Cohen, M. A. Matar, J. Zohar and H. Cohen, in Sleep and Combat-Related Post Traumatic Stress Disorder, Springer, 2018, pp. 127-143.

46. J. P. Herman and J. G. Tasker, Frontiers in endocrinology, 2016, 7, 137.

47. R. A. Morey, C. C. Haswell, S. R. Hooper and M. D. De Bellis, Neuropsychopharmacology, 2016, 41, 791. 
48. S. J. van Rooij, J. S. Stevens, T. D. Ely, R. Hinrichs, V. Michopoulos, S. J. Winters, Y. E. Ogbonmwan, J. Shin, N. R. Nugent and L. A. Hudak, Biological psychiatry, 2018, 84, 106115.

49. S. Van Rooij, M. Kennis, R. Sjouwerman, M. Van Den Heuvel, R. Kahn and E. Geuze, Psychological medicine, 2015, 45, 2737-2746.

50. M. D. Nelson and A. M. Tumpap, CNS spectrums, 2017, 22, 363-372.

51. M. A. Penzo, V. Robert, J. Tucciarone, D. De Bundel, M. Wang, L. Van Aelst, M. Darvas, L. F. Parada, R. D. Palmiter and M. He, Nature, 2015, 519, 455.

52. D. T. Hsu, G. J. Kirouac, J.-K. Zubieta and S. Bhatnagar, Frontiers in behavioral neuroscience, $2014, \mathbf{8}, 73$.

53. G. J. Kirouac, Neuroscience \& Biobehavioral Reviews, 2015, 56, 315-329.

54. E. Z. Millan, Z. Ong and G. P. McNally, in Progress in Brain Research, eds. T. Calvey and W. M. U. Daniels, Elsevier, 2017, vol. 235, pp. 113-137.

55. K. Zhou and Y. Zhu, Pharmacological Research, 2019, 142, 70-76.

56. G. Z. Réus, H. M. Abelaira, A. L. Maciel, M. A. B. dos Santos, A. S. Carlessi, A. V. Steckert, G. K. Ferreira, S. D. De Prá, E. L. Streck, D. S. Macêdo and J. Quevedo, Metabolic Brain Disease, 2015, 30, 545-553.

57. T. G. Oliveira, R. B. Chan, F. V. Bravo, A. Miranda, R. R. Silva, B. Zhou, F. Marques, V. Pinto, J. J. Cerqueira, G. Di Paolo and N. Sousa, Molecular Psychiatry, 2015, 21, 80.

58. P. R. Zoladz, M. Fleshner and D. M. Diamond, Psychoneuroendocrinology, 2012, 37, 1531-1545.

59. O. M. Ogundele, P. J. Ebenezer, C. C. Lee and J. Francis, Neuroscience, 2017, 353, 147165.

60. C. B. Wilson, P. J. Ebenezer, L. D. McLaughlin and J. Francis, PLoS One, 2014, 9, e89104.

61. C. B. Wilson, L. D. McLaughlin, P. J. Ebenezer, A. R. Nair, R. Dange, J. G. Harre, T. L. Shaak, D. M. Diamond and J. Francis, Frontiers in behavioral neuroscience, 2014, 8, 256.

62. R. M. Spiers, J. Marzi, E. M. Brauchle, S. E. Cross, R. H. Vaughan, P. A. Bateman, S. J. Hughes, K. Schenke-Layland and P. R. Johnson, Acta biomaterialia, 2019, 99, 269-283.

63. A. Ditta, H. Nawaz, T. Mahmood, M. Majeed, M. Tahir, N. Rashid, M. Muddassar, A. AlSaadi and H. Byrne, Spectrochimica Acta Part A: Molecular and Biomolecular Spectroscopy, 2019, 221, 117173.

64. J. Marzi, E. M. Brauchle, K. Schenke-Layland and M. W. Rolle, Acta biomaterialia, 2019, 89, 193-205.

65. C. Molony, J. McIntyre, A. Maguire, R. Hakimjavadi, D. Burtenshaw, G. Casey, M. Di Luca, B. Hennelly, H. J. Byrne and P. A. Cahill, Biochimica et Biophysica Acta (BBA)Molecular Cell Research, 2018, 1865, 343-353.

66. M. Karas, H. Ehring, E. Nordhoff, B. Stahl, K. Strupat, F. Hillenkamp, M. Grehl and B. Krebs, Organic Mass Spectrometry, 1993, 28, 1476-1481.

67. G. Robichaud, K. P. Garrard, J. A. Barry and D. C. Muddiman, Journal of the American Society for Mass Spectrometry, 2013, 24, 718-721.

68. M. T. Bokhart, M. Nazari, K. P. Garrard and D. C. Muddiman, Journal of The American Society for Mass Spectrometry, 2017, 29, 8-16.

69. C. Krafft, L. Neudert, T. Simat and R. Salzer, Spectrochimica Acta Part A: Molecular and Biomolecular Spectroscopy, 2005, 61, 1529-1535.

70. L. Macala, R. Yu and S. Ando, Journal of lipid research, 1983, 24, 1243-1250.

71. M. A. Wells and J. C. Dittmer, Biochemistry, 1967, 6, 3169-3175. 
72. B. Schrader, Raman, infrared atlas of organic compounds, VCH-Verlag-Ges., 1989.

73. R. L. Frost, M. L. Weier, K. L. Erickson, O. Carmody and S. J. Mills, Journal of Raman Spectroscopy, 2004, 35, 1047-1055.

74. K. Ben Mabrouk, T. H. Kauffmann, H. Aroui and M. D. Fontana, Journal of Raman Spectroscopy, 2013, 44, 1603-1608.

75. L. Zhang, M. J. Henson and S. S. Sekulic, Analytica Chimica Acta, 2005, 545, 262-278.

76. K. C. Gordon and C. M. McGoverin, International journal of pharmaceutics, 2011, 417, 151-162.

77. S. E. Bohndiek, A. Wagadarikar, C. L. Zavaleta, D. Van de Sompel, E. Garai, J. V. Jokerst, S. Yazdanfar and S. S. Gambhir, Proceedings of the National Academy of Sciences, 2013, 110, 12408-12413.

78. M. Noponen, M. Sanfilipo, K. Samanich, H. Ryer, G. Ko, B. Angrist, A. Wolkin, E. Duncan and J. Rotrosen, Biological Psychiatry, 1993, 34, 641-649.

79. T. Cajka and O. Fiehn, TrAC Trends in Analytical Chemistry, 2014, 61, 192-206.

80. D. Y. Bang and M. H. Moon, Journal of Chromatography A, 2013, 1310, 82-90.

81. G. Tan, Z. Lou, W. Liao, X. Dong, Z. Zhu, W. Li and Y. Chai, Molecular BioSystems, 2012, 8, 548-556.

82. C. Zhu, A. Dane, G. Spijksma, M. Wang, J. Van der Greef, G. Luo, T. Hankemeier and R. J. Vreeken, Journal of Chromatography A, 2012, 1220, 26-34.

83. I. Ottestad, S. Hassani, G. I. Borge, A. Kohler, G. Vogt, T. Hyötyläinen, M. Orešič, K. W. Brønner, K. B. Holven and S. M. Ulven, PLoS One, 2012, 7.

84. H. Nygren, T. Seppänen-Laakso, S. Castillo, T. Hyötyläinen and M. Orešič, in Metabolic Profiling, Springer, 2011, pp. 247-257.

85. H. Nygren, P. Pöhö, T. Seppänen-Laakso, U. Lahtinen, M. Oresic and T. Hyötyläinen, $L C$ GC Europe, 2013, 26, 142-148.

86. I. Kaya, W. Michno, D. Brinet, Y. Iacone, G. Zanni, K. Blennow, H. Zetterberg and J. r. Hanrieder, Analytical chemistry, 2017, 89, 4685-4694.

87. C. G. Pick, The Consequences of Exposure to Mission-Related Shock Waves Upon Cognitive Potential, TEL-AVIV UNIV (ISRAEL), 2010.

88. S. N. Jackson, H.-Y. J. Wang and A. S. Woods, Analytical chemistry, 2005, 77, 4523-4527.

89. D. G. Harper, J. E. Jensen, C. Ravichandran, Y. Sivrioglu, M. Silveri, D. V. Iosifescu, P. F. Renshaw and B. P. Forester, The American Journal of Geriatric Psychiatry, 2014, 22, 499-509.

90. W. S. Davidson, A. Jonas, D. F. Clayton and J. M. George, Journal of Biological Chemistry, 1998, 273, 9443-9449.

91. F. Mesa-Herrera, L. Taoro-González, C. Valdés-Baizabal, M. Diaz and R. Marín, International journal of molecular sciences, 2019, 20, 3810.

92. S. N. Jackson, H.-Y. J. Wang and A. S. Woods, Journal of the American Society for Mass Spectrometry, 2007, 18, 17-26.

93. M. Jain, S. Ngoy, S. A. Sheth, R. A. Swanson, E. P. Rhee, R. Liao, C. B. Clish, V. K. Mootha and R. Nilsson, American Journal of Physiology-Endocrinology and Metabolism, 2014, 306, E854-E868.

94. S. Sonnino and V. Chigorno, Biochimica et Biophysica Acta (BBA)-Reviews on Biomembranes, 2000, 1469, 63-77.

95. V. Martín, N. Fabelo, G. Santpere, B. Puig, R. Marín, I. Ferrer and M. Díaz, Journal of Alzheimer's Disease, 2010, 19, 489-502. 
96. I. Kracun, H. Rosner, V. Drnovsek, Z. Vukelic, C. Cosovic, M. Trbojevic-Cepe and M. Kubat, Neurochemistry international, 1992, 20, 421-431.

97. T. G. Oliveira, R. B. Chan, F. V. Bravo, A. Miranda, R. R. Silva, B. Zhou, F. Marques, V. Pinto, J. J. Cerqueira and G. Di Paolo, Molecular psychiatry, 2016, 21, 80-88. 


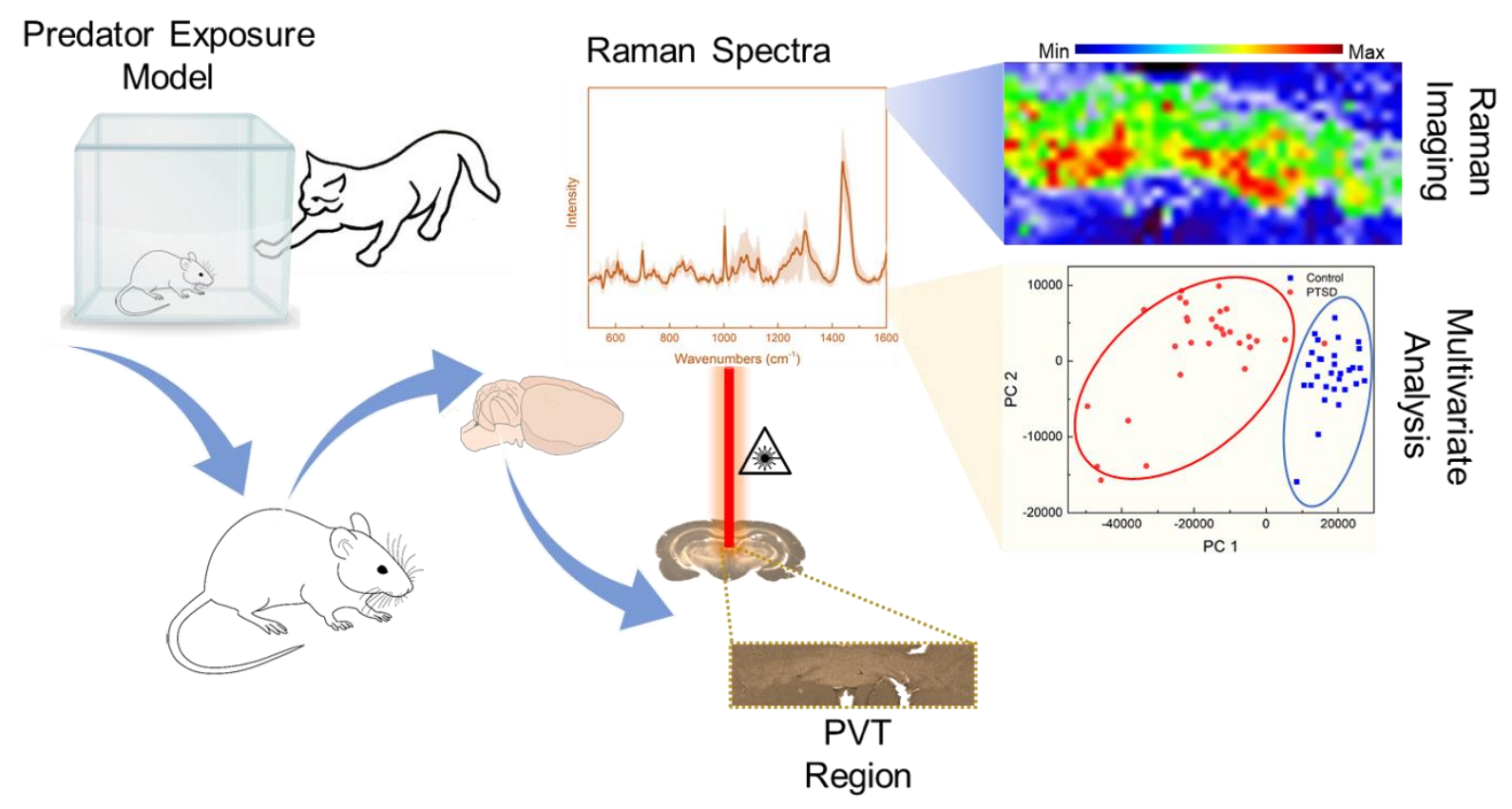

Figure 1. Schematic showing the design of experiment. Brain tissue from control and PTSD rats was obtained. Multivariate statistical analyses were performed on the Raman spectra and Raman images to identify the biochemical changes in the paraventricular nucleus of the thalamus (PVT) region of the brain. 


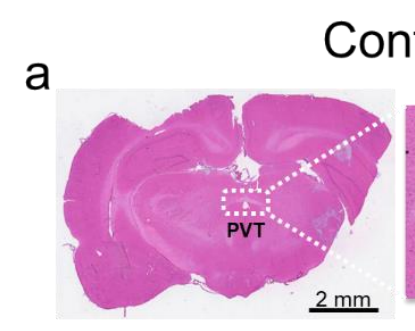

Control
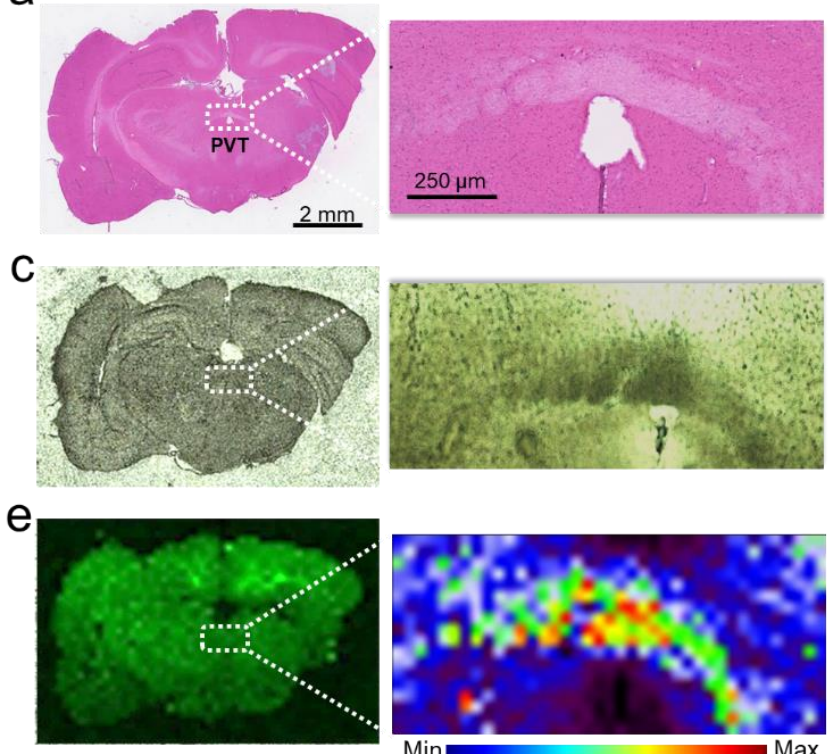

b
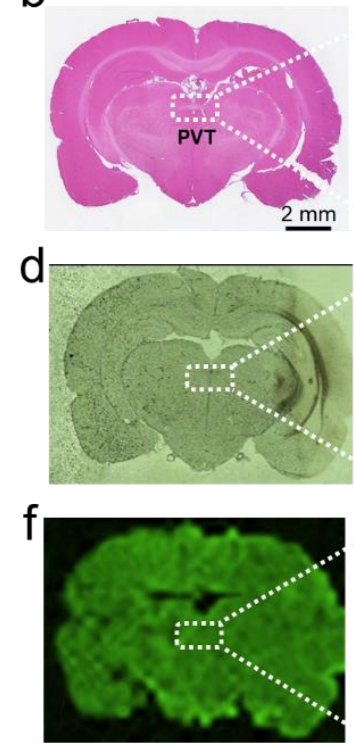

PTSD
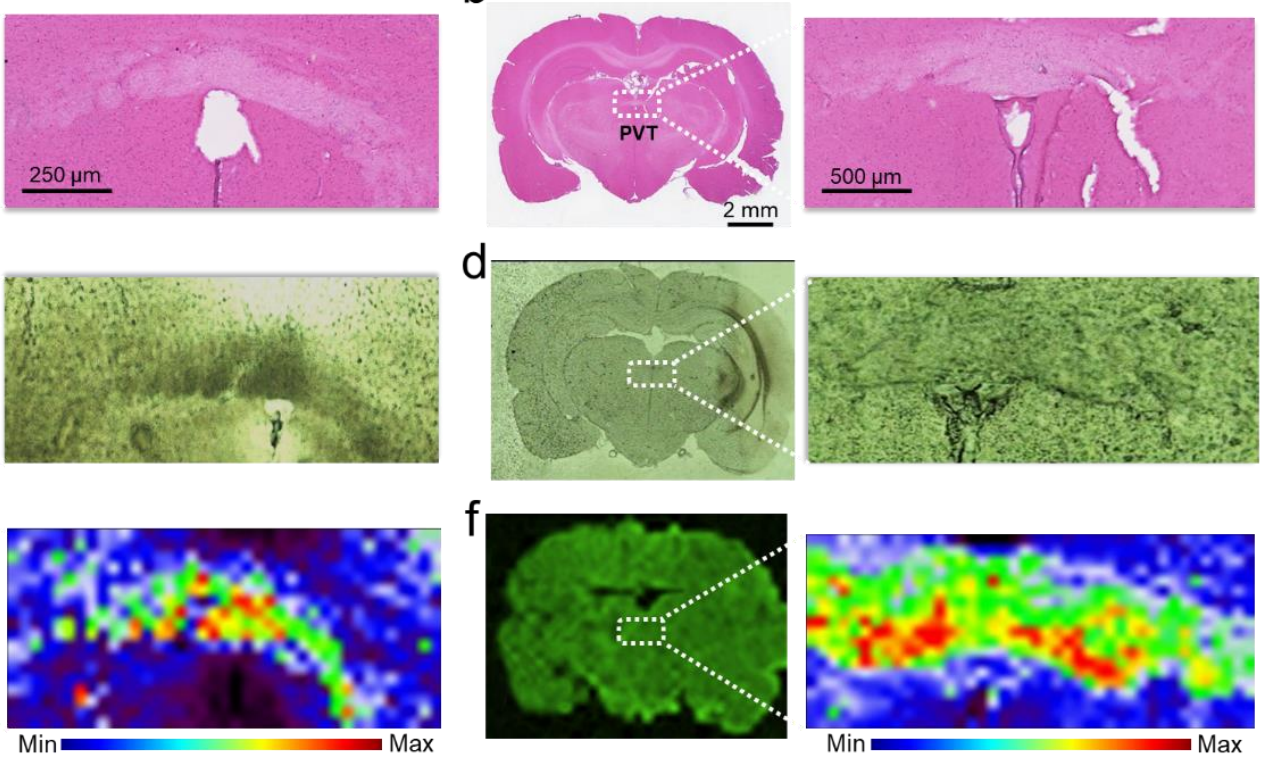

Figure 2. Histology and Raman imaging of brain tissues. H\&E stained brain tissue of (a) Control, and (b) PTSD rat. The inset zoomed image shows the paraventricular nucleus of the thalamus (PVT) region. Bright field image of the brain tissue for the (c) Control, (d) PTSD rat. The corresponding Raman imaging map at $1002 \mathrm{~cm}^{-1}$ collected using a $\lambda=785 \mathrm{~nm}$ laser is shown for the (e) Control, and (f) PTSD rat. The inset shows the distribution of phenylalanine within the PVT region. 
a

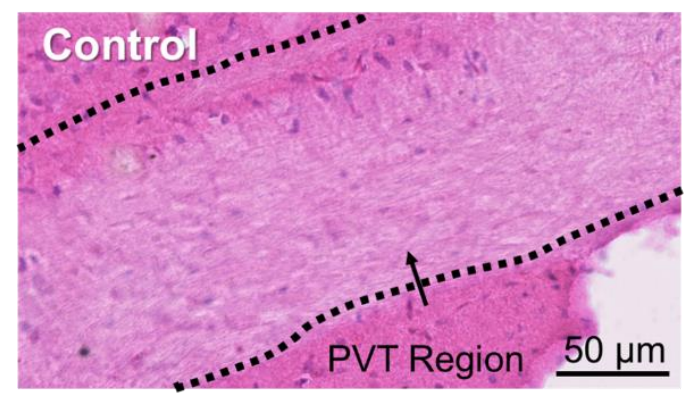

C
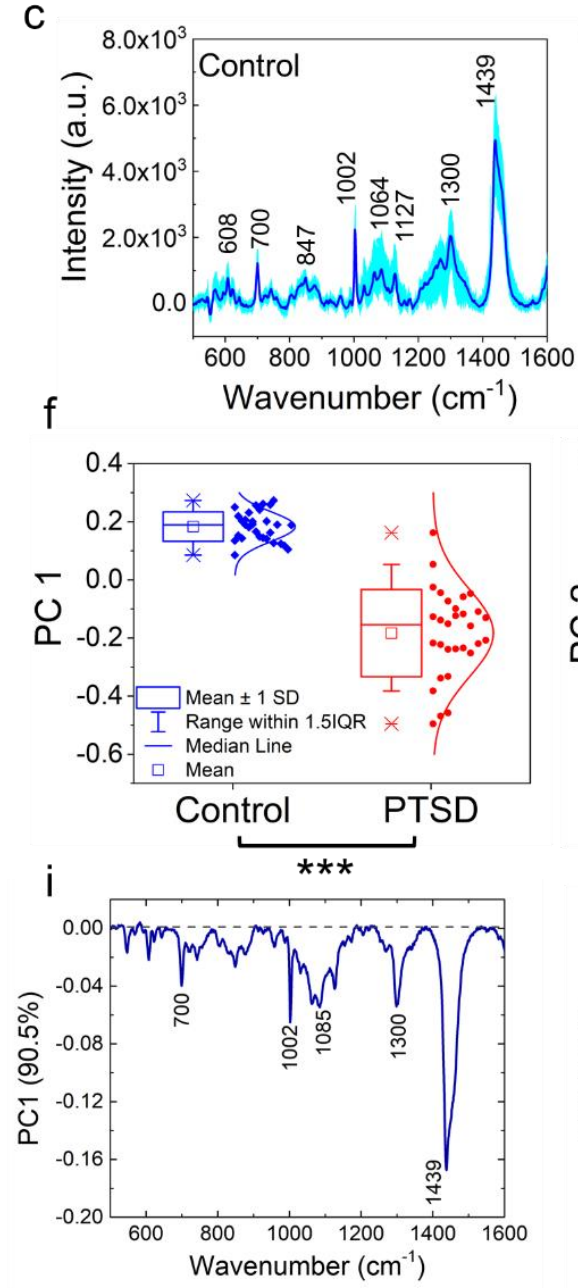

d

g b
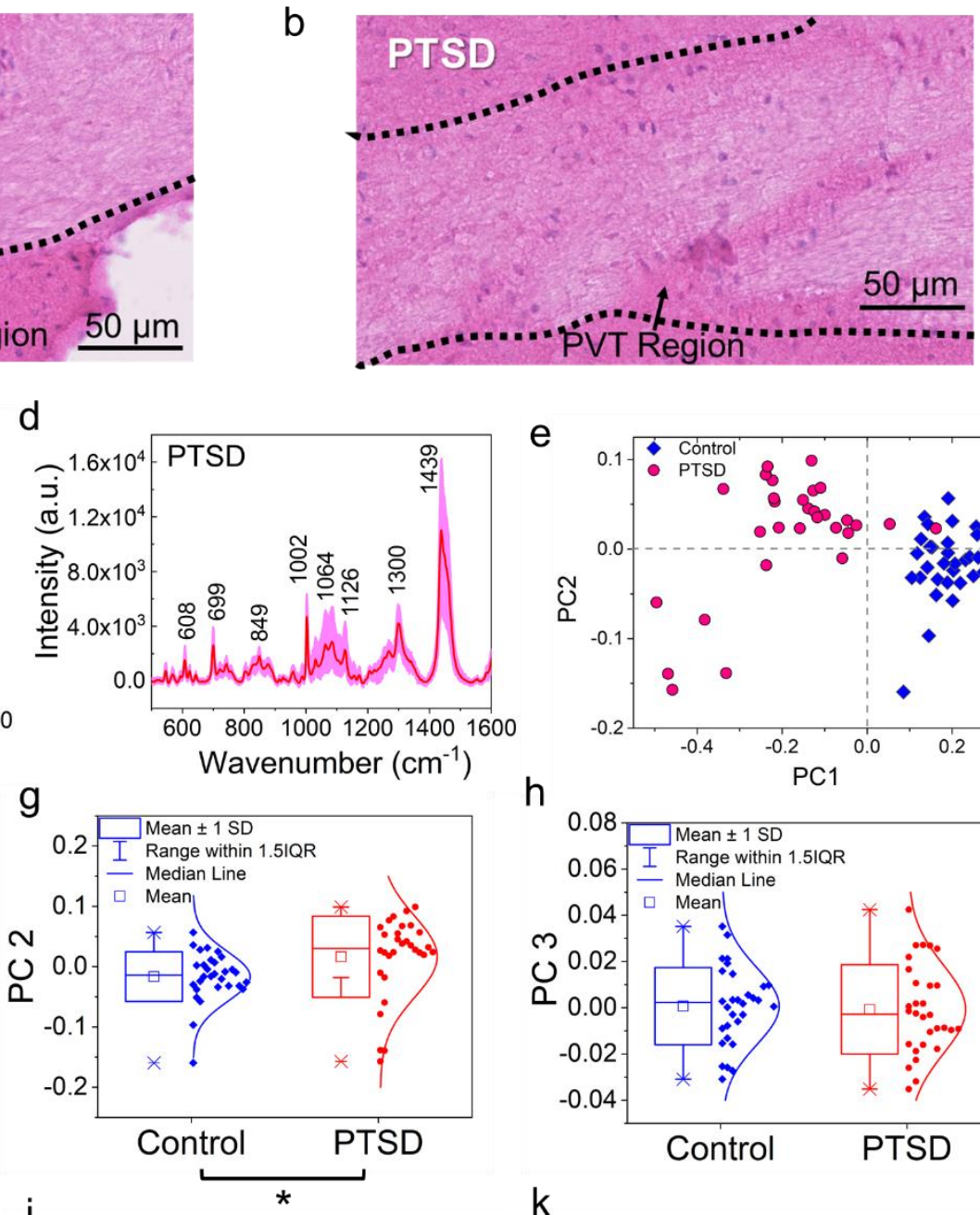

h

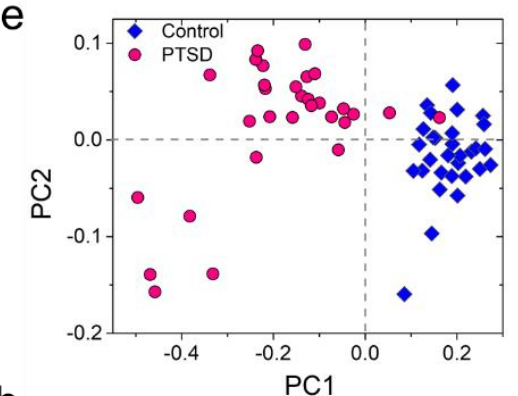

$\mathrm{h}$
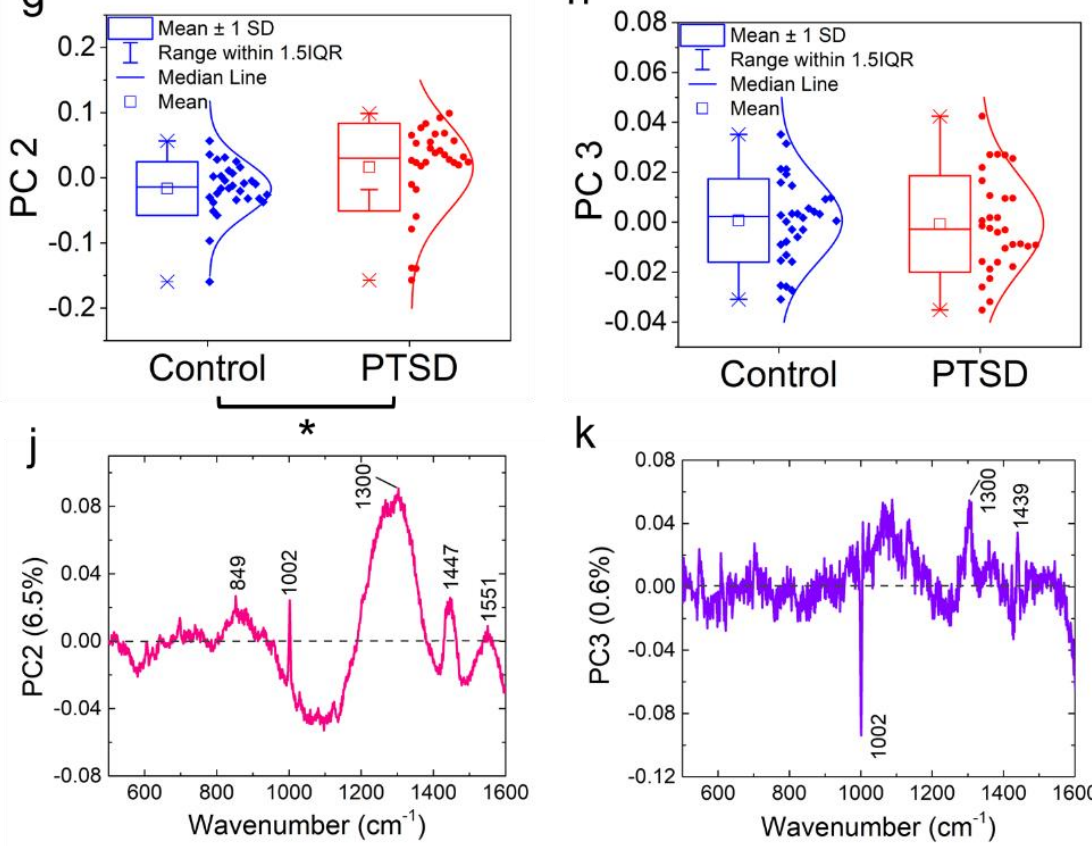

k

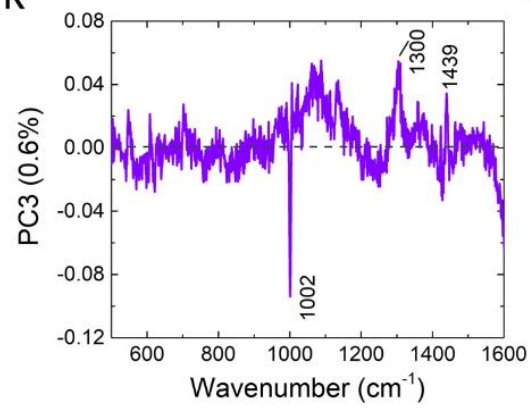

Figure 3. Raman spectroscopy, multivariate analysis, and associated histology images of the

PVT region. Representative H\&E image of the PVT region for the (a) Control, (b) PTSD rat. The corresponding mean Raman spectra of (c) Control, (d) PTSD brain tissue. The standard deviation of all the spectra is shown as shaded color for each sample. (e) PCA score plot showing the separation between PTSD and control tissue sample along PC 1. The distribution of score values 
for (f) PC 1, (g) PC 2, and (h) PC 3. The corresponding loading plots highlighting the significant peaks responsible for the separation in the score plot for (i) PC 1, (j) PC 2, and (k) PC 3 . $* P<$ $0.05, * * P<0.01, * * * P<0.001$, total number of spectra, $\mathrm{n}=30$.

a

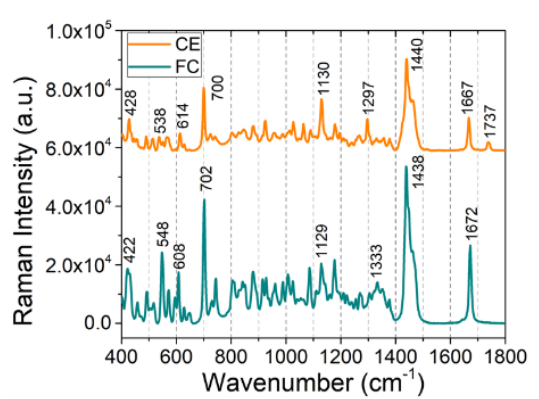

d

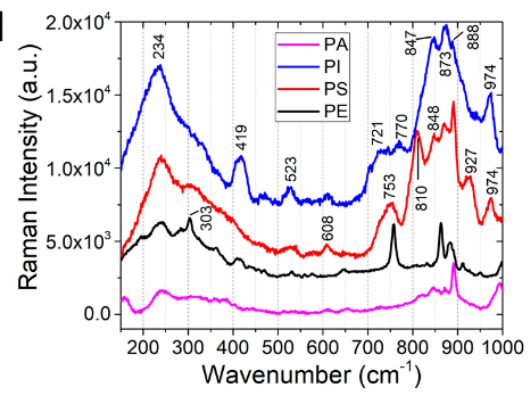

g

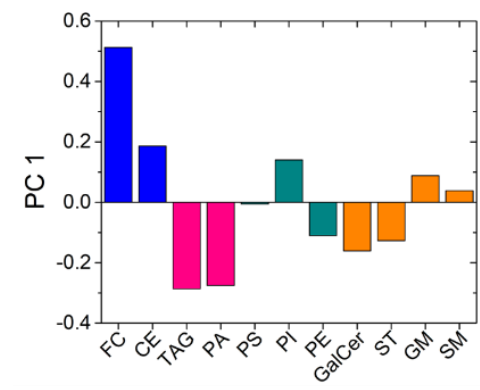

j

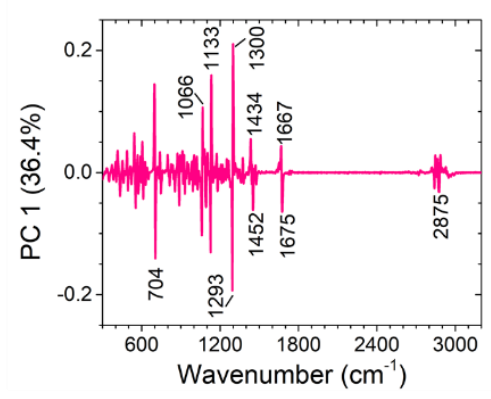

b
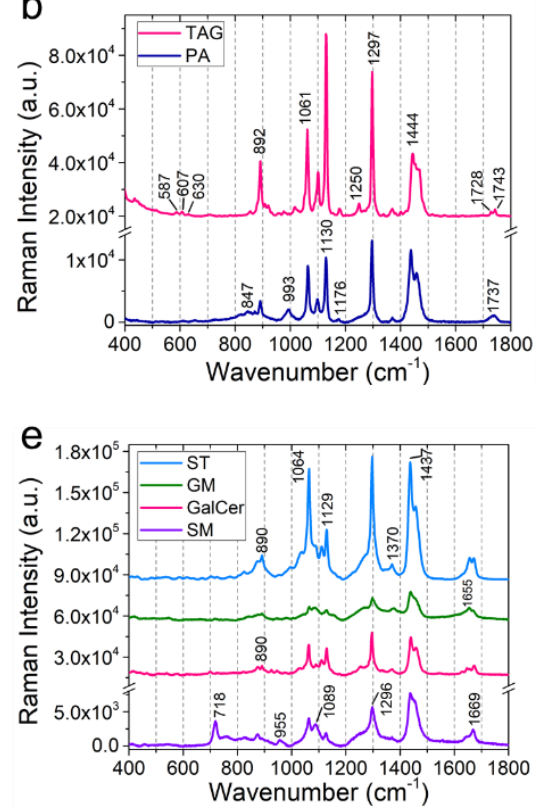

h
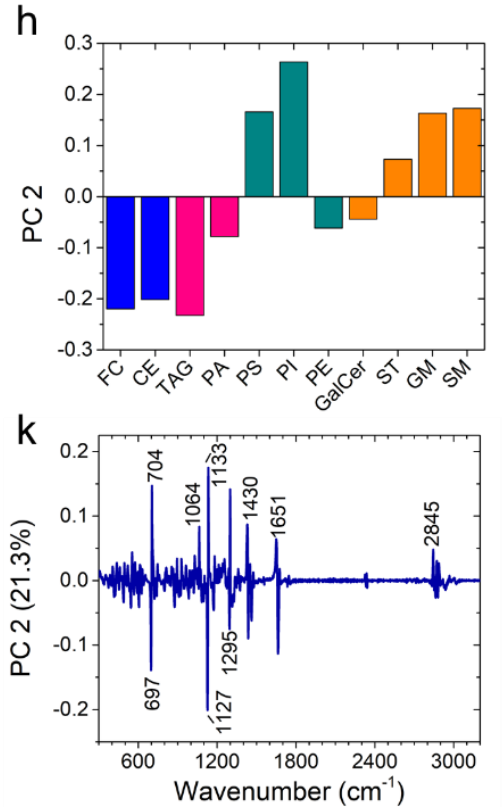

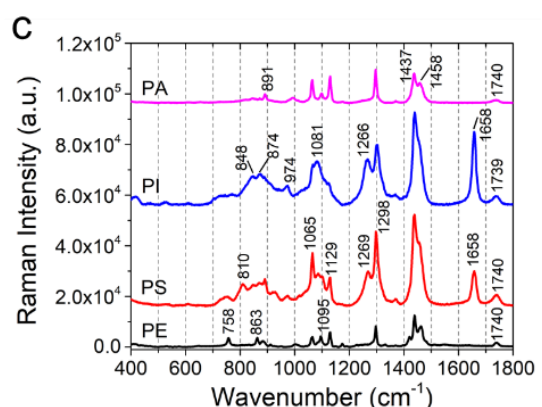

f

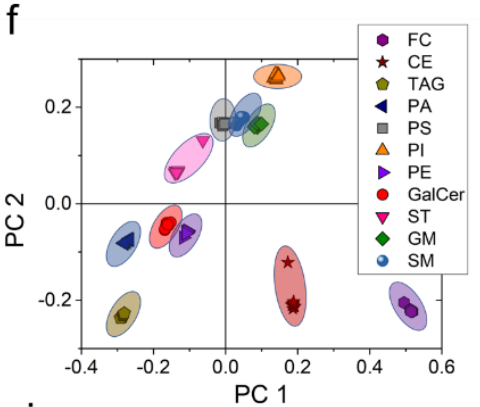

i
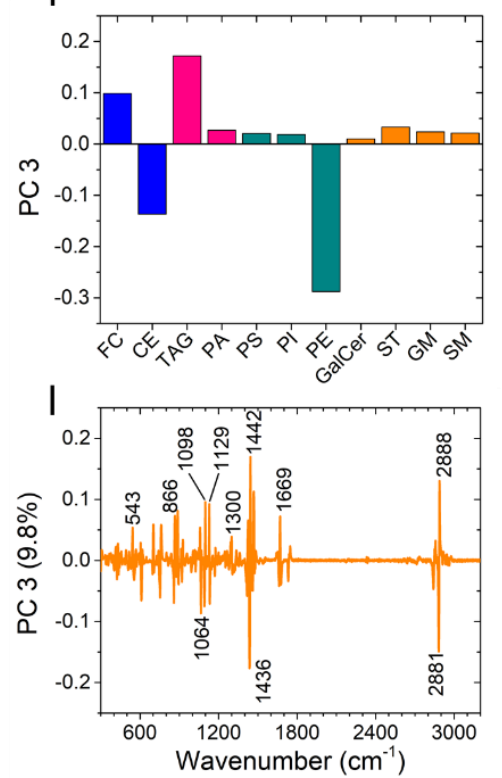

Figure 4. Raman spectra and multivariate analysis for the reference standards of eleven most abundant lipids in brain. The Raman spectra are grouped into lipid class such as sterol lipids, 
glycerolipids, phospholipids, and sphingolipids. Comparison of Raman spectra from 400 to $1800 \mathrm{~cm}^{-1}$ for (a) cholesterol (free cholesterol, FC), and cholesteryl palmitate (cholesterol ester, CE); (b) glyceryl palmitate (triacylglyceride, TAG), and phosphatidic acid (PA); (c) phosphatidylinositol (PI), phosphatidylserine (PS), and phosphatidylethanolamine (PE); (d) the Raman spectra of phospholipids in the low wavenumber range of $200-1000 \mathrm{~cm}^{-1}$; (e) comparison of Raman spectra from 400 to $1800 \mathrm{~cm}^{-1}$ for sulfatide (ST), ganglioside (GM), galactocerebroside (GalCer), and sphingomyelin (SM). (f) Multivariate analysis showing one-way PCA scatter plot of the first two principal components. The first derivate of the raw Raman spectra was performed to construct the PCA score plot. $(\mathrm{g}, \mathrm{h}, \mathrm{i})$ and $(\mathrm{j}, \mathrm{k}, \mathrm{l})$ their corresponding loading plots are shown for PC 1, PC 2, and PC 3, respectively. 

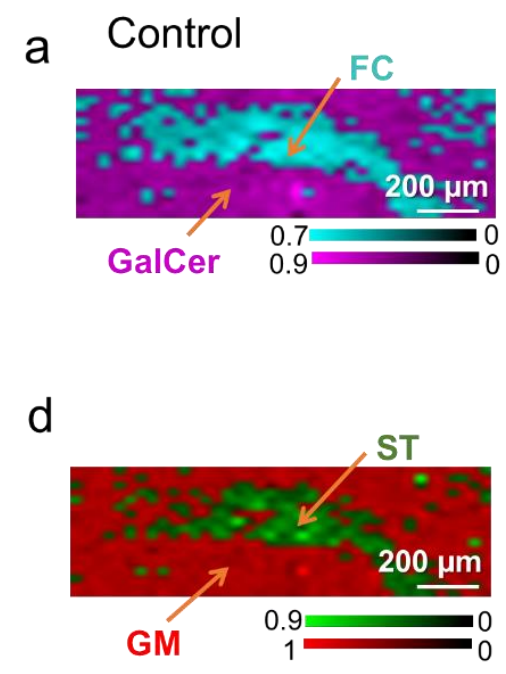

g

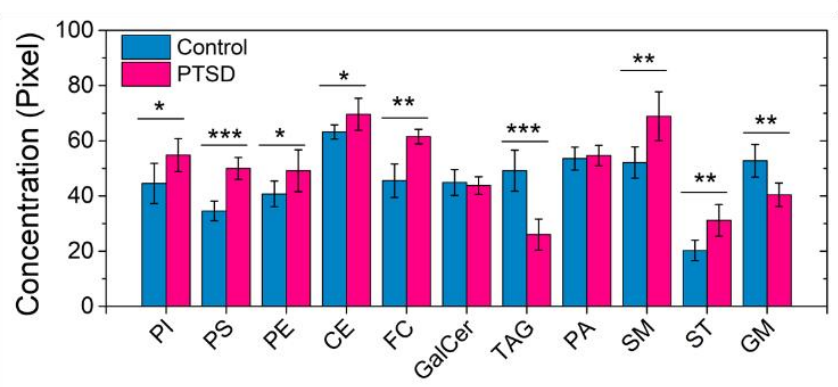

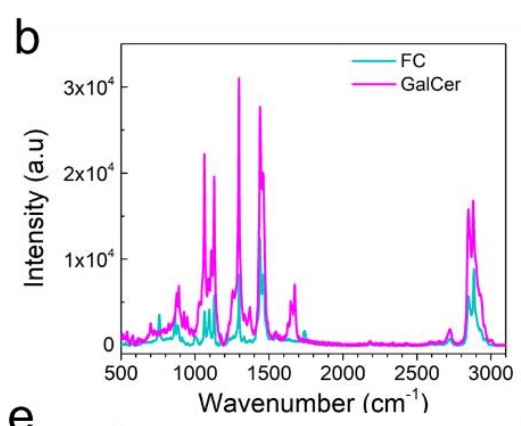

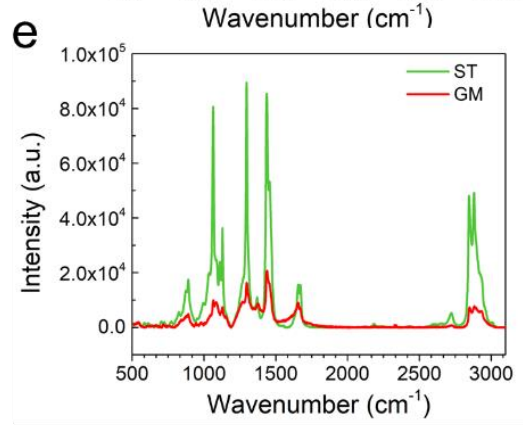

C

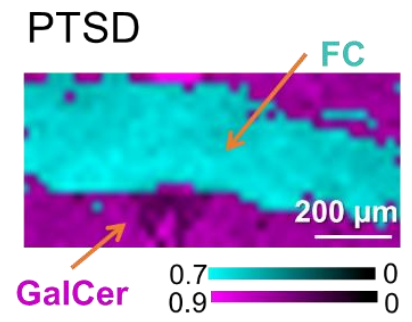

f

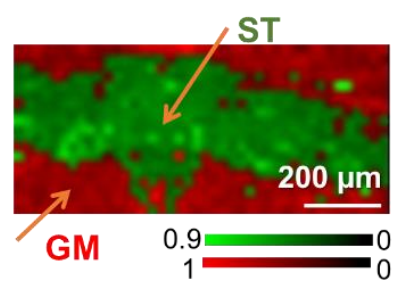

$\mathrm{h}$

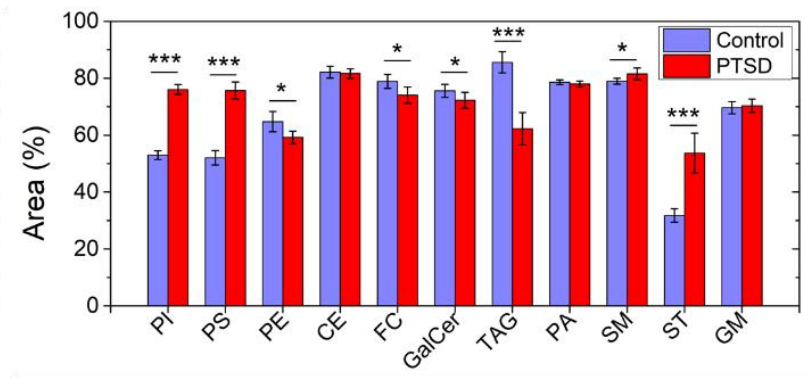

Figure 5. Comparison of DCLS Raman spectroscopy images of the lipids from the respective

PVT region. DCLS Raman heat maps of cholesterol (FC) and galactocerebroside (GalCer) plotted from the signature Raman spectra for (a) Control, (c) PTSD brain sample. The Raman spectra are shown in (b); (d-e) Raman heat maps and Raman spectra of sulfatide (ST) and ganglioside (GM); Average of relative concentration of lipids within PVT region calculated from the (g) pixel intensity, (h) areal distribution of the Raman images. Statistical significance were calculated using Student's paired t-test, with a two-tailed distribution. $* P<0.05, * * P<0.01, * * * P<0.001, \mathrm{n}=5$ per group. 

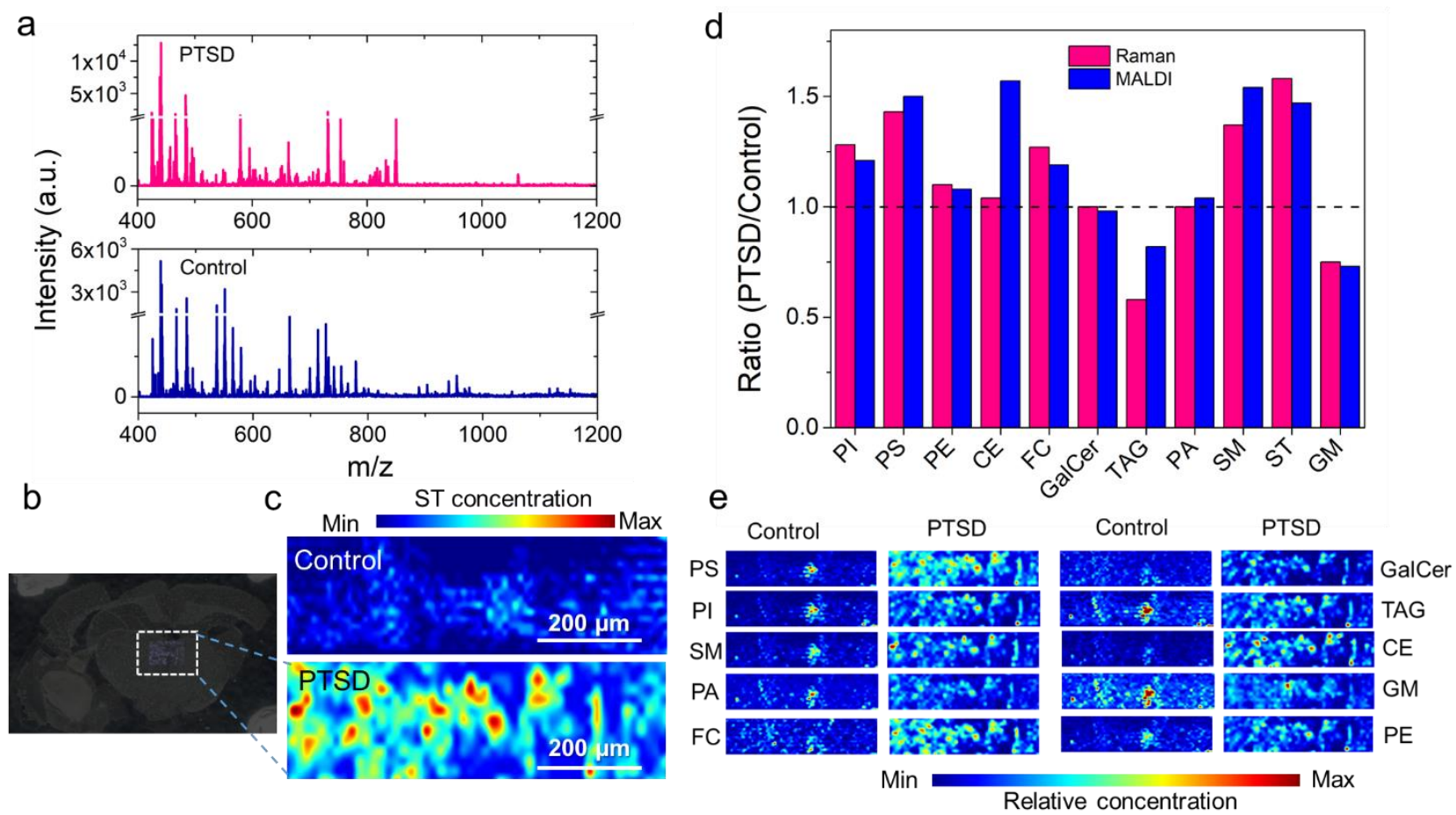

Figure 6. Mass spectra and MALDI images obtained from the PVT region of the brain using the positive ion mode. (a) Representative MALDI mass spectra acquired from the control (blue), and PTSD (pink) brain sample. (b) Bright field optical image, and (c) False color two-dimensional MALDI-MS image of sulfatides in control (top) and PTSD (bottom) sample (ST 38:1, $\mathrm{m} / z$ 890.5); (d) Ratio of PTSD to control calculated from the Raman images using the mean value of pixel intensity (pink bars) for different lipids. The corresponding PTSD to control ratio calculated from each MALDI-MS images (blue bars) are also compared with the Raman ratio. Ratio, $\mathrm{R}=1$ indicates no change, $\mathrm{R}>1$ indicates increase, and $\mathrm{R}<1$ indicates decrease of specific lipids in PTSD samples compared to the control samples. (e) Representative MALDI-MS images of selected lipid species in control and PTSD brain samples. The images were constructed at the following mass peak: phosphatidylserine (PS 34:2, $\mathrm{m} / \mathrm{z}$ 760.5), phosphatidylinositol (PI 31:3, $\mathrm{m} / \mathrm{z}$ 829.4), sphingomyelin (SM 33:0-O2, $\mathrm{m} / \mathrm{z}$ 729.5), phosphatidic acid (PA 32:1, $\mathrm{m} / \mathrm{z}$ 647.5), cholesterol (FC 24:5, m/z 401.2), galactocerebroside (GalCer 34:1-O2, m/z 722.5), 
triacylglyceride (TAG 50:9, m/z 817.6), cholesterol ester (CE 18:3, m/z 669.6), ganglioside (GM or Hex(4)-HexNAc-Fuc-Cer 36:1-O2, m/z 1563.9), phosphatidylethanolamine (PE 36:6, m/z 736.5); the species number equal the total length and number of double bonds of both acyl chains; mass error $<50 \mathrm{ppm}$.

\section{Abstract Graphic}

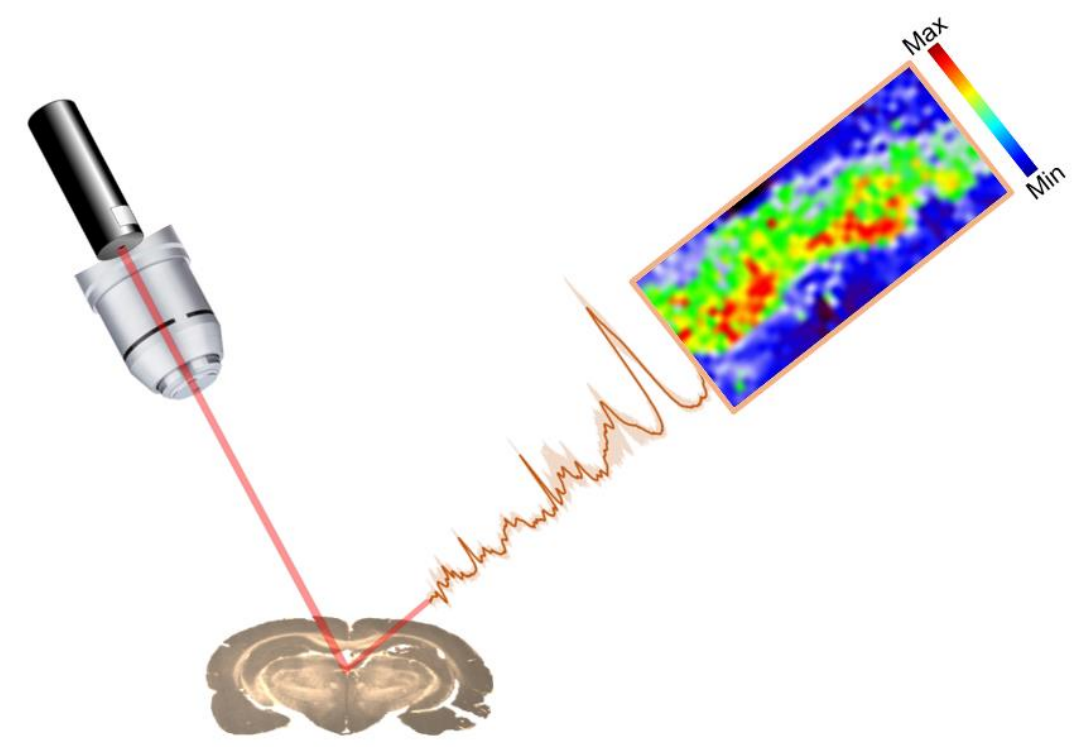

